\title{
Synthesis of Staphylococcus aureus lipoteichoic acid derivatives for determining the minimal structural requirements for cytokine induction
}

\author{
Ignacio Figueroa-Perez, ${ }^{\mathrm{a}}$ Andreas Stadelmaier, ${ }^{\mathrm{a}}$ Susanne Deininger, ${ }^{\mathrm{b}}$ Sonja von Aulock, ${ }^{\mathrm{b}}$ \\ Thomas Hartung ${ }^{\mathrm{b}}$ and Richard R. Schmidt ${ }^{\mathrm{a}, *}$ \\ ${ }^{a}$ Fachbereich Chemie, Universität Konstanz, Fach M 725, D-78457 Konstanz, Germany \\ ${ }^{\mathrm{b}}$ Fachbereich Biologie, Universität Konstanz, D-78457 Konstanz, Germany
}

\begin{abstract}
For the investigation of the minimal structural requirements for cytokine induction, Staphylococcus aureus lipoteichoic acid derivatives with two, three, four, and five glycerophosphate backbone moieties, carrying each a D-alanyl residue, were needed. Based on two different glycerophosphate building blocks and 6b-O-phosphitylated gentiobiosyl diacylglycerol the desired target molecules (compounds 1-4) could be readily obtained and provided for biological studies.
\end{abstract}

(c) 2006 Elsevier Ltd. All rights reserved.

Keywords: Bacteria; Gram-positive; Lipoteichoic acid; Synthesis; Modifications

\section{Introduction}

During infection, the recognition of conserved bacterial structures called pathogen-associated molecular patterns occurs via receptor recognition on immune cells and leads to the activation of the innate immune system resulting in the release of a variety of cytokines. Lipopolysaccharides (LPS) have been known as the most important conserved bacterial structures of Gram-negative bacteria inducing cytokine release for more than 50 years. ${ }^{1}$ Immune recognition takes place by the binding of LPS to the toll-like receptor 4 (TLR4) involving also other co-factors. ${ }^{2-4}$

The immunostimulatory component of Gram-positive bacteria was not clear for a long time, although a structural counterpart of LPS called lipoteichoic acid (LTA) was found in the bacterial membrane; this LTA is also an amphiphilic molecule with a lipid anchor and a gen-

\footnotetext{
* Corresponding author. Tel.: +49 7531 882538; fax: +49 7531

883135; e-mail: Richard.Schmidt@uni-konstanz.de
}

erally negatively charged glycerophosphate backbone. An improved preparation procedure applied to the isolation of LTA from Staphylococcus aureus led to biologically active LTA, ${ }^{5-7}$ whose structure could be assigned by NMR and MS data. ${ }^{5,8}$ The receptor for LTA recognition is TLR $2^{9}$ accompanied by the co-factors TLR6, ${ }^{7}$ $\mathrm{CD} 14,{ }^{10,11}$ and CD36. ${ }^{12}$

Modifications of the LTA structure gave information on the prerequisites for the induction of cytokine release. For instance, a complete deacylation led to less active material and selective removal of the D-alanyl residues from the glycerophosphate backbone which strongly reduced the immunostimulatory potency. ${ }^{8}$ This result indicated that the lipid anchor and also the D-alanyl residues are essential for the immunostimulatory potency. The chemical synthesis of a truncated LTA, based on the native LTA structure of $S$. aureus, confirmed these results. ${ }^{13,14}$ To determine the key components for immune cell activation, several LTA derivatives were synthesized starting from a molecule with two lipid anchors ( $=$ two gentiobiosyl-diacylglycerol anchors) and a backbone with six glycerophosphate units substituted 

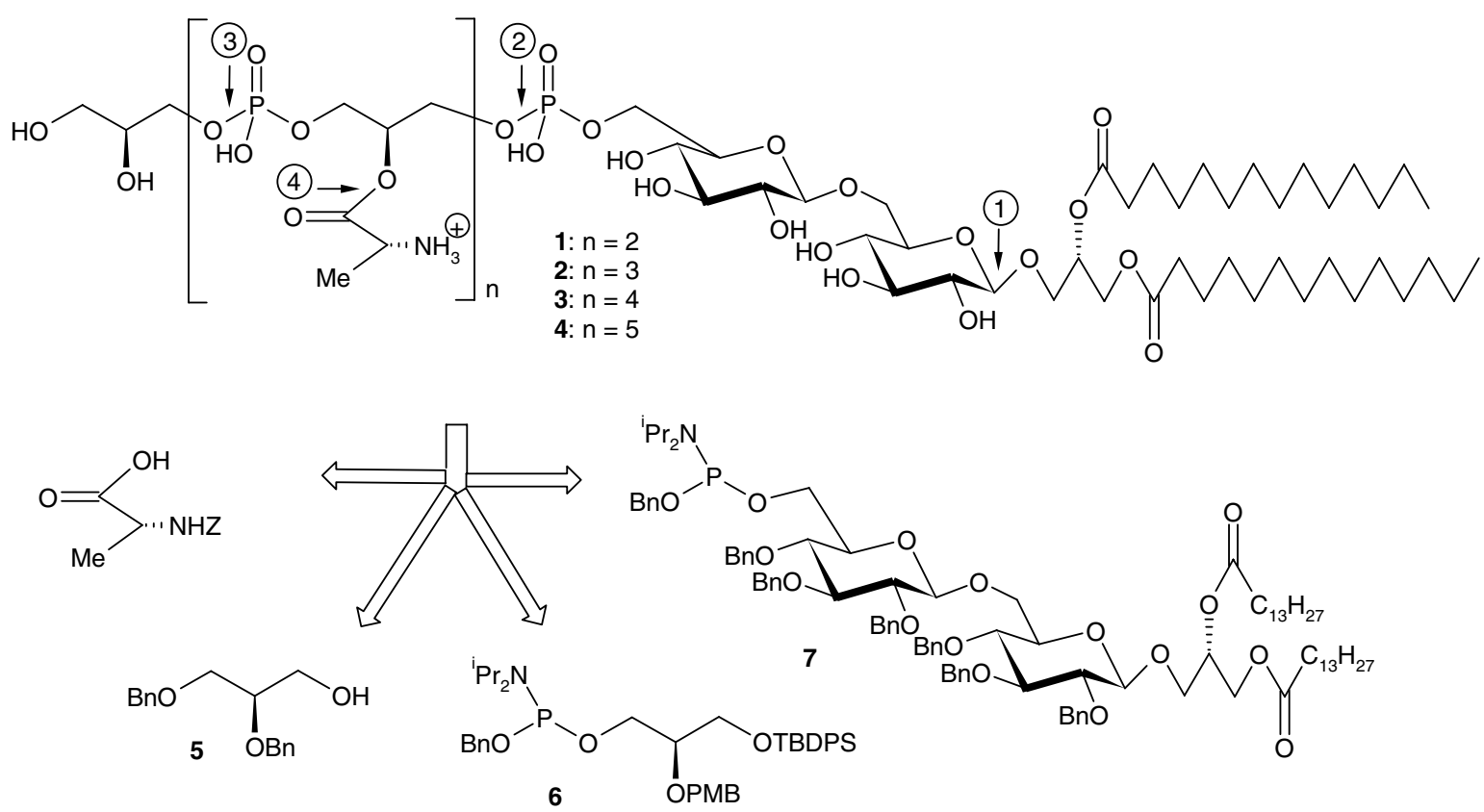

Scheme 1. Retrosynthetic scheme for target molecules 1-4.

by four D-alanine residues and one $N$-acetyl-D-glucosamine residue which was more potent than natural LTA. ${ }^{15}$ Neither the absence of the gentiobiose residue nor the loss of $N$-acetyl-D-glucosamine altered the ability of LTA with one lipid anchor to induce cytokine release; only replacement of $\mathrm{D}$-alanine by $\mathrm{L}$-alanine blunted the cytokine-inducing potency. ${ }^{16,17}$ These results approximated the crucial pattern required for the immune recognition of LTA and prompted us to synthesize further LTA derivatives with a reduced structure down to the synthetic anchor, namely gentiobiosyl-diacylglycerol, in order to determine the minimal structural requirements for cytokine induction. For these studies compounds 1-4 (Scheme 1) were needed. The synthesis of these compounds is described in this paper.

\section{Results and discussion}

The retrosynthesis of compounds $\mathbf{1 - 4}$ is shown in Scheme 1. Disintegrations (1)-(4) lead to building blocks 5-7 and to $N$-benzyloxy-carbonyl (Z)-protected D-alanine. These building blocks consider the presence of gentiobiosyl diacylglycerol, $O$-(D-alanyl)-glycerol, and 2-Ononsubstituted glycerol residues, respectively, and their sequence specific linkage via mixed phosphorous diester bonds. Also the most important aspect, the hydrolytic lability of the D-alanyl residues, which are readily cleaved at $\mathrm{pH} 8.5,{ }^{13}$ is taken into account: as temporary protecting group for building block $\mathbf{6}$, the $p$-methoxylbenzyl (PMB) group is chosen which can be selectively cleaved after completion of the backbone synthesis. Following the attachment of D-alanyl residues with Z-pro- tected D-alanine and then a complete O-debenzylation will provide the target molecules.

The synthesis of the previously designed building blocks 5-7 and also the sequence specific synthesis of oligomers 8-10 (Scheme 2) has been already reported. ${ }^{14,15,17}$ From gentiobiose building block $7^{15}$ and glycerophosphate oligomer $\mathbf{8},{ }^{17}$ possessing the required protecting group for regioselective chain extension and following D-alanyl residue attachment, the synthesis of target molecule 1 could be readily accomplished. Ligation of $\mathbf{7}$ and $\mathbf{8}$ in the presence of tetrazole and then oxidation with tert-butylhydroperoxide gave phosphate linked intermediate 13, which contains the backbone of the target molecule with $n=2$. The treatment of $\mathbf{1 3}$ with ceric(IV) ammonium nitrate $(\mathrm{CAN})^{18}$ liberated two of the glycerol hydroxy groups affording compound 14. Attachment of the Z-protected D-alanyl residue was performed with excess Z-protected D-alanine in the presence of (benzotriazol-1-yloxy)tripyrrolidinophosphonium hexyfluorophosphate (PyBOP) ${ }^{19} / N$-methylimidazole (Me-Im) as condensing agent to give the fully protected target molecule 15. Hydrogenolysis with Pearlman's catalys $\mathrm{t}^{20}$ in a mixture of dichloromethane/ methanol/water gave the desired final product $\mathbf{1}$ with two D-alanyl residues.

Similarly, from 7 and $\mathbf{9}^{17}$ or $\mathbf{1 0} 0^{17}$ compounds $\mathbf{1 6}$ and 19 , respectively, were obtained, which were transformed into the partially deprotected compounds $\mathbf{1 8}$ and $\mathbf{2 1}$ and then into target molecules $\mathbf{2}$ and $\mathbf{3}$, having three or four D-alanyl residues. For the synthesis of target molecule $\mathbf{4}$, compound 10 had to be chain extended with building block $6 .{ }^{17}$ The activation of $\mathbf{6}$ with tetrazole led to the desired phosphite intermediate which on oxidation with 

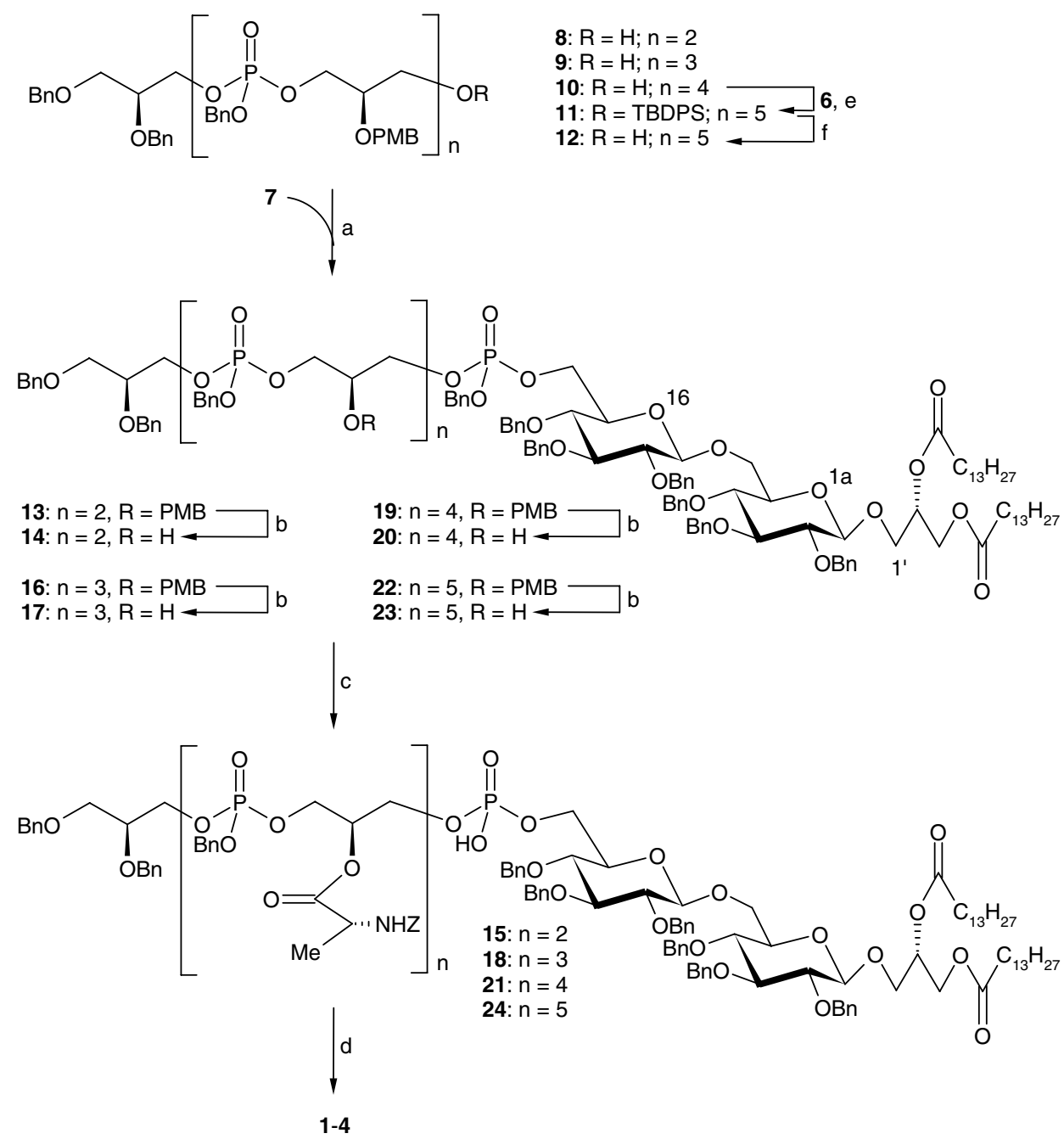

Scheme 2. Synthesis of target molecules 1-4. Reagents and conditions: (a) Tetrazole, $\mathrm{CH}_{2} \mathrm{Cl}_{2} ; t-\mathrm{BuO}_{2} \mathrm{H}(\mathbf{1 3}: 73 \% ; 16: 84 \% ; 19: 74 \% ; \mathbf{2 2}: 67 \%)$; (b) $\mathrm{CAN}, \mathrm{MeCN} / \mathrm{Tol} / \mathrm{H}_{2} \mathrm{O},-10^{\circ} \mathrm{C} \rightarrow \mathrm{rt}\left(14: 72 \% ; 17: 87 \%\right.$; 20: 85\%; 23: 80\%); (c) (Z)-D-Ala, PyBOP, Me-Im, $\mathrm{CH}_{2} \mathrm{Cl}_{2}(15: 92 \%$; 18: 56\%; 21: 69\%; 24: $58 \%$ ); (d) $\mathrm{Pd}(\mathrm{OH})_{2}, \mathrm{H}_{2}, \mathrm{CH}_{2} \mathrm{Cl}_{2} / \mathrm{MeOH} / \mathrm{H}_{2} \mathrm{O}(1: 32 \% ; 2: 41 \% ; 3: 26 \% ; 4: 28 \%)$; (e) tetrazole, $\mathrm{CH}_{2} \mathrm{Cl}_{2} ; t-\mathrm{BuO}_{2} \mathrm{H}(83 \%)$; (f) TBAF, THF (80\%).

tert-butyl hydroperoxide furnished the phosphate $\mathbf{1 1}$ having six glycerol and five phosphate residues. Desilylation with tetrabutylammonium fluoride (TBAF) in tetrahydrofuran led to intermediate 12; further chain extension with gentiobiosyl diacylglycerol derivative 7 furnished compound 22. Cleavage of the PMB groups $(\rightarrow \mathbf{2 3})$, then the attachment of five Z-protected D-alanyl residues $(\rightarrow \mathbf{2 4})$, and final deprotection afforded target molecule 4.

In summary, the previously designed building blocks for the synthesis of $S$. aureus LTA proved to be highly versatile, thus permitting the preparation of variously modified LTAs. This cassette containing about six building blocks laid the basis for successful structureactivity relationship studies. ${ }^{21}$

The biological studies with compounds 1-4 and some previously prepared $S$. aureus LTA modifications revealed the following: ${ }^{21}$ The synthetic lipid anchor alone was not sufficient to induce cytokine release, however, the addition of three unsubstituted glycerophosphate backbone units exhibits this activity. The TLR2-dependent activation was amplified about 10-fold by substitution with at least two D-alanyl residues (compound 2) - but not by L-alanyl residues. Hence, with compounds 1-4 the minimal structural requirement for LTA pattern recognition by immune cells could be defined.

\section{Experimental}

\subsection{General methods}

Solvents were dried according to standard procedures. NMR spectroscopic measurements were performed at $22{ }^{\circ} \mathrm{C}$ with a Bruker DRX600 and Bruker AC250 
instruments. TMS or the resonances of the deuterated solvents were used as an internal standard. $\mathrm{CDCl}_{3}$ $(\delta=7.24 \mathrm{ppm})$ was used as an external standard; $85 \%$ of phosphoric acid was used as an external standard for ${ }^{31} \mathrm{P}$ spectra. MALDI mass spectra were recorded with a Kratos Kompact Maldi II spectrometer; 2,5dihydroxybenzoic acid (DHB) or p-nitroaniline and $\mathrm{NaI}$ were used as matrices for positive measurements, and trihydroxyacetophenone (THAP) was used as the matrix for negative mode measurements. Optical rotations were measured with a Perkin-Elmer polarimeter $241 / \mathrm{MS}$ in a $1-\mathrm{dm}$ cell at $22^{\circ} \mathrm{C}$. Thin layer chromatography (TLC) was performed on E. Merck Silica Gel $60 \mathrm{~F}_{254}$ plastic plates. The compounds were visualized by a treatment with a solution of $\left(\mathrm{NH}_{4}\right)_{6} \mathrm{Mo}_{7} \mathrm{O}_{24} \cdot 4 \mathrm{H}_{2} \mathrm{O}$ $(20 \mathrm{~g})$ and $\mathrm{Ce}\left(\mathrm{SO}_{4}\right)_{2}(0.4 \mathrm{~g})$ in $10 \% \mathrm{H}_{2} \mathrm{SO}_{4}(400 \mathrm{~mL})$. Flash chromatography was performed on J.T. Baker Silica Gel $60(0.040-0.063 \mathrm{~mm})$ at a pressure of 0.3 bar. Target molecules were purified by hydrophobic interaction chromatography on octyl-sepharose as the stationary phase and as the elution phase was used as a gradient of propanol $(15-60 \%)$ in $0.1 \mathrm{M}$ ammonium acetate buffer $(\mathrm{pH}=4.8)$.

3.2. (2,3-Di-O-benzyl-sn-glycero)-benzyloxyphosphoryl(2-O-(4-methoxybenzyl)-sn-glycero)-benzyloxyphosphoryl-(2-O-(4-methoxybenzyl)-sn-glycero)-benzyloxyphosphoryl-(2-O-(4-methoxybenzyl)-sn-glycero)-benzyloxyphosphoryl-(2-O-(4-methoxybenzyl)-sn-glycero)-benzyloxyphosphoryl-(2-O-(4-methoxybenzyl)-1-O-tert-butyldiphenylsilyl-sn-glycerol) (11)

A mixture of the glycerol moiety $\mathbf{1 0}^{17}(1.63 \mathrm{~g}$, $0.94 \mathrm{mmol})$ and phosphite amide $\mathbf{6}^{17}(0.78 \mathrm{~g}, 1.2$ equiv) was co-evaporated with dry $\mathrm{CH}_{2} \mathrm{Cl}_{2}$ and dried for $1 \mathrm{~h}$ in high vacuum. The reaction mixture was dissolved in $\mathrm{CH}_{2} \mathrm{Cl}_{2}(50 \mathrm{~mL})$, and tetrazole was added $(132 \mathrm{mg}$, $1.88 \mathrm{mmol}$, dried previously $1 \mathrm{~h}$ in a high vacuum). The reaction mixture was stirred for $1.5 \mathrm{~h}$ at $\mathrm{rt}$ (TLC petroleum ether/EtOAc 2:1, $\left.1 \% \mathrm{NEt}_{3}, R_{\mathrm{f}} 0.74\right)$ and $t$-BuOOH $(1.5 \mathrm{~mL})$ was added. After $20 \mathrm{~min}$, the reaction mixture was diluted with $\mathrm{CH}_{2} \mathrm{Cl}_{2}$ and washed with satd $\mathrm{NaHCO}_{3}$ solution, the organic phase was dried over $\mathrm{MgSO}_{4}$ and the solvent was evaporated under diminished pressure. Flash chromatography (2:1 toluene-acetone ) yielded phosphate $11(1.82 \mathrm{~g}, 83 \%)$ as colorless oil. TLC (1:1 toluene-acetone): $R_{\mathrm{f}} 0.64$, 0.59 . $[\alpha]_{\mathrm{D}}-3.4$ (c 1, $\left.\mathrm{CHCl}_{3}\right) .{ }^{1} \mathrm{H}$ NMR $(250 \mathrm{MHz}$, $\left.\mathrm{CDCl}_{3}\right): \delta 1.02(\mathrm{~s}, 9 \mathrm{H}, t-\mathrm{Bu}), 3.48-3.58(\mathrm{~m}, 2 \mathrm{H}, 18-\mathrm{H})$, 3.59-3.81 (m, 23H, 1-H, 2-H, 5-H, 8-H, 11- H, 14- , 17-H, OMe $), 3.90-4.35(\mathrm{~m}, 20 \mathrm{H}, 3-\mathrm{H}, 4-\mathrm{H}, 6-\mathrm{H}$, 7-H, 9-H, 10-H, 12-H, 13-H, 15-H, 16-H), 4.39-4.52 $\left(\mathrm{m}, 12 \mathrm{H}, \mathrm{CH}_{2}-\mathrm{Ph}\right), 4.58-4.66\left(\mathrm{~m}, 2 \mathrm{H}, \mathrm{CH}_{2}-\mathrm{Ph}\right)$, 4.91-5.06 (m, $\left.10 \mathrm{H}, \mathrm{POCH}_{2}-\mathrm{Ph}\right), 6.71-6.85(\mathrm{~m}, 10 \mathrm{H}$, $\mathrm{Ph}_{\mathrm{PMB}}$ ), 7.10-7.47, 7.59-7.69 (m, 55H, Ph). MALDIMS: $\mathrm{m} / \mathrm{z} 2355.8[\mathrm{M}+\mathrm{Na}]^{+}$. Anal. Calcd for $\mathrm{C}_{123} \mathrm{H}_{143} \mathrm{O}_{33} \mathrm{P}_{5} \mathrm{Si}$
(2332.4): C, 63.34; H, 6.18. Found: C, 63.17; H, 6.28 .

3.3. (2,3-Di-O-benzyl-sn-glycero)-benzyloxyphosphoryl(2-O-(4-methoxybenzyl)-sn-glycero)-benzyloxyphosphoryl-(2-O-(4-methoxybenzyl)-sn-glycero)-benzyloxyphosphoryl-(2-O-(4-methoxybenzyl)-sn-glycero)-benzyloxyphosphoryl-(2-O-(4-methoxybenzyl)-sn-glycero)-benzyloxyphosphoryl-(2-O-(4-methoxybenzyl)-sn-glycerol) (12)

Phosphate 11 (1.8 g, $0.77 \mathrm{mmol}$ ) was dissolved in THF $(40 \mathrm{~mL})$ and treated with $1 \mathrm{M}$ solution of TBAF $(0.93 \mathrm{~mL}, 1.2$ equiv), the reaction mixture was stirred for $30 \mathrm{~min}$ at $\mathrm{rt}$. The reaction mixture was diluted with EtOAc and washed with satd $\mathrm{NH}_{4} \mathrm{Cl}$ solution and water, the organic phase was dried over $\mathrm{MgSO}_{4}$ and the solvent was evaporated under diminished pressure. Purification by flash silica gel (1:1 toluene-acetone) yielded compound $\mathbf{1 2}(1.3 \mathrm{~g}, 80 \%)$ as a colorless syrup. TLC (1:1 toluene-acetone): $R_{\mathrm{f}} 0.36 .[\alpha]_{\mathrm{D}}-1.2(c 1$, $\left.\mathrm{CHCl}_{3}\right) .{ }^{1} \mathrm{H}$ NMR $\left(250 \mathrm{MHz}, \mathrm{CDCl}_{3}\right): \delta 2.83-3.01(\mathrm{br}$ $\mathrm{s}, 1 \mathrm{H}, \mathrm{OH}), 3.49-3.84(\mathrm{~m}, 25 \mathrm{H}, 1-\mathrm{H}, 2-\mathrm{H}, 5-\mathrm{H}, 8-\mathrm{H}$, 11- H, 14- H, 17-H, 18-H, OMe), 3.91-4.26 (m, $20 \mathrm{H}$, 3-H, 4-H，6-H，7-H，9-H，10-H，12-H，13-H，15-H, 16-H), $4.40-4.65\left(\mathrm{~m}, 14 \mathrm{H}, \mathrm{CH}_{2}-\mathrm{Ph}\right), 4.92-5.09(\mathrm{~m}$, $10 \mathrm{H}, \mathrm{POCH}{ }_{2}-\mathrm{Ph}$ ), 6.72-6.89 (m, 10H, $\left.\mathrm{Ph}_{\mathrm{PMB}}\right), 7.13-7.44$ (m, 45H, Ph). MALDIMS: $m / z 2117.6[\mathrm{M}+\mathrm{Na}]^{+}$. Anal. Calcd for $\mathrm{C}_{107} \mathrm{H}_{125} \mathrm{O}_{33} \mathrm{P}_{5} \cdot 1 / 2 \mathrm{H}_{2} \mathrm{O}$ (2103.0): $\mathrm{C}, 61.11 ; \mathrm{H}$, 6.04. Found: C, 61.00; H, 6.16.

3.4. (2,3-Di-O-benzyl-sn-glycero)-benzyloxyphosphoryl(2-O-(4-methoxybenzyl)-sn-glycero)-benzyloxyphosphoryl-(2-O-(4-methoxybenzyl)-sn-glycero)-benzyloxyphosphoryl-6- $O$-(2,3,4-tri- $O$-benzyl- $\beta$-D-glucopyranosyl)-6- $O$ (2,3,4-tri- $O$-benzyl- $\beta$-D-glucopyranosyl)-1,2-di- $O$-myristoyl-sn-glycerol (13)

Compound $\mathbf{8}^{17}(250 \mathrm{mg}, 0.250 \mathrm{mmol})$ and phosphite amide $7^{15}$ (403 mg, 1.3 equiv) were co-evaporated each with $10 \mathrm{~mL}$ of dry $\mathrm{CH}_{2} \mathrm{Cl}_{2}$ and dried $1 \mathrm{~h}$ in high vacuum. Phosphite amide 7 was dissolved in $10 \mathrm{~mL}$ of dry $\mathrm{CH}_{2} \mathrm{Cl}_{2}$ and was added, under argon atmosphere, to compound 8; tetrazole ( $36 \mathrm{mg}$, 2 equiv, dried previously $1 \mathrm{~h}$ in high vacuum) was also added. The reaction mixture was stirred at $\mathrm{rt}$ under argon atmosphere. After $70 \mathrm{~min}$ were added dropwise $0.7 \mathrm{~mL}$ of $t \mathrm{BuOOH}$, and the reaction mixture was stirred for another $35 \mathrm{~min}$. $\mathrm{CH}_{2} \mathrm{Cl}_{2}$ was added to dilute and the mixture was washed with satd $\mathrm{NaHCO}_{3}$ solution, the organic phase was dried over $\mathrm{MgSO}_{4}$ and the solvent removed under diminished pressure. Purification by flash chromatography (petroleum ether/EtOAc 2:1) gave compound $\mathbf{1 3}$ (464 $\mathrm{mg}, 73 \%$ ) as a colorless syrup, which was stored at $-20{ }^{\circ} \mathrm{C}$. TLC (petroleum ether/EtOAc 1:1): $R_{\mathrm{f}} 0.53$ and 0.63. $[\alpha]_{\mathrm{D}}+3.4\left(c 1, \mathrm{CHCl}_{3}\right) .{ }^{1} \mathrm{H} \mathrm{NMR}(600 \mathrm{MHz}$, $\left.\mathrm{CDCl}_{3}\right): \delta 0.88(\mathrm{t}, 6 \mathrm{H}, \mathrm{Me}), 1.08-1.34\left(\mathrm{~m}, 40 \mathrm{H}, \mathrm{CH}_{2^{-}}\right.$ 
chain), 1.46-1.59 (m, 4H, $\left.\mathrm{COCH}_{2} \mathrm{CH}_{2} \mathrm{R}\right), 2.17-2.26(\mathrm{~m}$, $\left.4 \mathrm{H}, \mathrm{COCH}_{2} \mathrm{CH}_{2} \mathrm{R}\right), 3.35(\mathrm{~m}, 2 \mathrm{H}, 2 \mathrm{a}-\mathrm{H}, 5 \mathrm{a} / \mathrm{b}-\mathrm{H}), 3,38$ (m, 1H, 1'-H), 3.39 (m, 2H, 2b-H, 5a/b-H), 3.47-3.49 $(\mathrm{m}, 2 \mathrm{H}, 4 \mathrm{a}-\mathrm{H}, 4 \mathrm{~b}-\mathrm{H}), 3.52(\mathrm{~m}, 2 \mathrm{H}, 9-\mathrm{H}), 3.60(\mathrm{~m}, 2 \mathrm{H}$, $3 \mathrm{a}-\mathrm{H}, 3 \mathrm{~b}-\mathrm{H}), 3.61$ (m, 1H, 6a-H), 3.66 (m, 2H, 2-H, 5$\mathrm{H}), 3.69(\mathrm{~m}, 6 \mathrm{H}, \mathrm{OMe}), 3.73(\mathrm{~m}, 1 \mathrm{H}, 8-\mathrm{H}), 3.86(\mathrm{~m}$, $\left.1 \mathrm{H}, 1^{\prime}-\mathrm{H}\right), 3.97-4.05(\mathrm{~m}, 8 \mathrm{H}, 1-\mathrm{H}, 3-\mathrm{H}, 4-\mathrm{H}, 6-\mathrm{H})$, $4.08\left(\mathrm{~m}, 2 \mathrm{H}, 7-\mathrm{H}, 3^{\prime}-\mathrm{H}\right), 4.14(\mathrm{~m}, 1 \mathrm{H}, 6 \mathrm{a}-\mathrm{H}), 4.16(\mathrm{~m}$, $\left.1 \mathrm{H}, 3^{\prime}-\mathrm{H}\right), 4.18$ (m, 2H, 7-H, 6b-H), 4.23 (m, 1H, 1a$\mathrm{H}), 4.27(\mathrm{~m}, 1 \mathrm{H}, 6 \mathrm{~b}-\mathrm{H}), 4.43(\mathrm{~m}, 1 \mathrm{H}, 1 \mathrm{~b}-\mathrm{H}), 4.44-4.93$ (m, 20H, $\left.\mathrm{CH}_{2} \mathrm{Ph}\right), 4.95-5.04\left(\mathrm{~m}, 6 \mathrm{H}, \mathrm{POC} \mathrm{H}_{2} \mathrm{Ph}\right), 5.09$ $\left(\mathrm{m}, 1 \mathrm{H}, 2^{\prime}-\mathrm{H}\right), 6.70-6.83\left(\mathrm{~m}, 4 \mathrm{H}, \mathrm{Ph}_{\mathrm{MPM}}\right), 7.08-7.37$ $(\mathrm{m}, 59 \mathrm{H}, \mathrm{Ph}) .{ }^{13} \mathrm{C} \mathrm{NMR}\left(150.9 \mathrm{MHz}, \mathrm{CDCl}_{3}\right): \delta 55.1$ (3C, OMe), 62.9 (1C, C-3'), 65.8 (4C, C-1, C-3, C-4, C-6), 66.5 (1C, C-6b), 67.0 (1C, C-8), 68.1 (1C, C-1'), 68.7 (1C, C-6a), 69.2 (1C, C-9), 69.5 (3C, $\left.\mathrm{POCH}_{2} \mathrm{Ph}\right)$, $69.8\left(1 \mathrm{C}, \mathrm{C}-2^{\prime}\right), 71.5-76.5\left(10 \mathrm{C}, \mathrm{CH}_{2} \mathrm{Ph}\right), 73.6$ (1C, C$5 \mathrm{a} / \mathrm{b}), 75.2$ (1C, C-4a/b), 75.4 (3C, C-2, C-5), 76.7 (1C, C-7), 77.1 (1C, C-4a/b), 78.0 (1C, C-5a/b), 81.9 (2C, C-2a, C-2b), 84.4 (2C, C-3a, C-3b), 103.6 (1C, C-1a), 104.0 (1C, C-1b). MALDIMS: $m / z 2552.3[\mathrm{M}+\mathrm{Na}]^{+}$. Anal. Calcd for $\mathrm{C}_{145} \mathrm{H}_{183} \mathrm{O}_{32} \mathrm{P}_{4}$ (2530.9): C, 68.81; H, 7.29. Found: C, 69.12; H, 7.53.

\section{5. (2,3-Di-O-benzyl-sn-glycero)-benzyloxyphosphoryl- (sn-glycero)-benzyloxyphosphoryl-(sn-glycero)-benzyl- oxyphosphoryl-6- $O$-(2,3,4-tri- $O$-benzyl- $\beta$-D-glucopyran- osyl)-6- $O$-(2,3,4-tri- $O$-benzyl- $\beta$-D-glucopyranosyl)-1,2- di- $O$-myristoyl-sn-glycerol (14)}

Compound 13 (450mg, $0.178 \mathrm{mmol}$ ) was dissolved in acetonitrile/toluene/water $(60: 3: 4,30 \mathrm{~mL})$ and cooled to $-10^{\circ} \mathrm{C}$. $\mathrm{Ce}\left(\mathrm{NH}_{4}\right)_{2}\left(\mathrm{NO}_{3}\right)_{6} \quad(0.776 \mathrm{~g}, 8$ equiv $)$ was added portionwise and the reaction mixture stirred for 20 min at $-10{ }^{\circ} \mathrm{C}$, the cooling bath was removed and the reaction mixture was stirred for another 30-40 min (TLC-monitoring). After this time the reaction mixture was diluted with EtOAc and washed with satd $\mathrm{NaHCO}_{3}$ solution, the organic phase was dried over $\mathrm{MgSO}_{4}$ and was evaporated under diminished pressure. A fast purification in silica gel (2.5:1 toluene-acetone) yielded compound 14 (294 mg, 72\%) as a colorless syrup, which was stored at $-20^{\circ} \mathrm{C}$. TLC (2:1 toluene-acetone): $R_{\mathrm{f}} 0.43, R_{\mathrm{f}}$ 0.52. $[\alpha]_{\mathrm{D}}+3.2\left(c 0.6, \mathrm{CHCl}_{3}\right) .{ }^{1} \mathrm{H} \mathrm{NMR}(600 \mathrm{MHz}$, $\left.\mathrm{CDCl}_{3}\right): \delta 0.78-0.94(\mathrm{t}, 6 \mathrm{H}, \mathrm{Me}), 1.08-1.38(\mathrm{~m}, 40 \mathrm{H}$, $\mathrm{CH}_{2}$-chain), 1.43-1.60 (m, 4H, $\left.\mathrm{COCH}_{2} \mathrm{CH}_{2} \mathrm{R}\right), 2.08-$ $2.26\left(\mathrm{~m}, 4 \mathrm{H}, \mathrm{COCH}_{2} \mathrm{CH}_{2} \mathrm{R}\right), 3.34(\mathrm{~m}, 1 \mathrm{H}, 2 \mathrm{a}-\mathrm{H}), 3.36$ (m, 1H, 5a/b-H), 3,39 (m, 3H, 5a/b, 1'-H, 2b-H), 3.48 $(\mathrm{m}, 2 \mathrm{H}, 4 \mathrm{a}-\mathrm{H}, 4 \mathrm{~b}-\mathrm{H}), 3.52(\mathrm{~m}, 2 \mathrm{H}, 9-\mathrm{H}), 3.59(\mathrm{~m}, 2 \mathrm{H}$, $3 \mathrm{a}-\mathrm{H}, 3 \mathrm{~b}-\mathrm{H}), 3.61(\mathrm{~m}, 1 \mathrm{H}, 6 \mathrm{a}-\mathrm{H}), 3.72(\mathrm{~m}, 1 \mathrm{H}, 8-\mathrm{H})$, $3.85\left(\mathrm{~m}, 1 \mathrm{H}, 1^{\prime}-\mathrm{H}\right), 3.96,4.06\left(\mathrm{~m}, 8 \mathrm{H}, \mathrm{CH}_{2 \mathrm{Glyc}}\right), 4.07$ $\left(\mathrm{m}, 1 \mathrm{H}, 3^{\prime}-\mathrm{H}\right), 4.09(\mathrm{~m}, 1 \mathrm{H}, 7-\mathrm{H}), 4.12(\mathrm{~m}, 1 \mathrm{H}, 6 \mathrm{a}-\mathrm{H})$, $4.16(\mathrm{~m}, 1 \mathrm{H}, 7-\mathrm{H}), 4.17(\mathrm{~m}, 1 \mathrm{H}, 6 \mathrm{~b}-\mathrm{H}), 4.18(\mathrm{~m}, 1 \mathrm{H}, 8-$ $\mathrm{H}), 4.24(\mathrm{~m}, 1 \mathrm{H}, 1 \mathrm{a}-\mathrm{H}), 4.30(\mathrm{~m}, 1 \mathrm{H}, 6 \mathrm{~b}-\mathrm{H}), 4.42(\mathrm{~m}$, $1 \mathrm{H}, 1 \mathrm{~b}-\mathrm{H}), 4.46-4.95\left(\mathrm{~m}, 16 \mathrm{H}, \mathrm{CH}_{2} \mathrm{Ph}\right), 5.02(\mathrm{~m}, 6 \mathrm{H}$, $\left.\mathrm{POCH}_{2} \mathrm{Ph}\right), 5.09\left(\mathrm{~m}, 1 \mathrm{H}, 2^{\prime}-\mathrm{H}\right), 7.08-7.44(\mathrm{~m}, 55 \mathrm{H}$,
Ph). $\left.{ }^{13} \mathrm{C} \mathrm{NMR} \mathrm{(150.9} \mathrm{MHz,} \mathrm{CDCl}_{3}\right): \delta 14.1$ (2C, Me), $24.8\left(2 \mathrm{C}, \mathrm{COCH}_{2} \mathrm{CH}_{2} \mathrm{R}\right), 22.7 / 29.7 / 31.9\left(20 \mathrm{C}, \mathrm{CH}_{2}-\right.$ chain), 34.1, $34.2\left(2 \mathrm{C}, \mathrm{COCH}_{2} \mathrm{R}\right), 63.8$ (1C, C-3'), 66.5 (1C, C-6b), 67.2 (1C, C-11), 68.1 (C-1', C-CH ( $_{2 \mathrm{Glyc}}$ ), 68.6 (1C, C-6a), 68.9 (1C, C-9), 69.7 (3C, $\left.\mathrm{POCH}_{2} \mathrm{Ph}\right)$, 69.8 (1C, C-2'), 72.2-76.0 (8C, $\left.\mathrm{CH}_{2} \mathrm{Ph}\right), 73.5$ (1C, C5a/b), 75.0 (1C, C-4a/b), 76.4 (1C, C-8), 76.9 (1C, C4a/b), 77.8 (1C, C-5a/b), 81.9 (2C, C-2a, C-2b), 84.4 (2C, C-3a, C-3b), 103.6 (1C, C-1a), 103.9 (1C, C-1b), 127.6-138.5 (C-Ph), 172.9/173.3 (2C, COOR). MALDIMS (positive Mode, Matrix $p$-Nitroaniline $+\mathrm{NaI}$, THF): $[\mathrm{M}+\mathrm{Na}]^{+}, m / z$ 2212.1; found: $m / z$ 2212.1. $\mathrm{C}_{129} \mathrm{H}_{167} \mathrm{O}_{30} \mathrm{P}_{3}$ (2290.6): calcd: C, 67.64; H, 7.35. Found: C, 67.37; H, 7.10.

\section{6. (2,3-Di-O-benzyl-sn-glycero)-benzyloxyphosphoryl- (2-O-( $\mathrm{N}$-benzyloxycarbonyl-D-alanyl)-sn-glycero)-benzyl- oxyphosphoryl-(2-O-( $\mathrm{N}$-benzyloxycarbonyl-D-alanyl)-sn- glycero)-benzyloxyphosphoryl-6-O-(2,3,4-tri- $O$-benzyl- $\beta$-D-glucopyranosyl)-6- $O$-(2,3,4-tri- $O$-benzyl- $\beta$-D-gluco- pyranosyl)-1,2-di- $O$-myristoyl-sn-glycerol (15)}

Compound 14 (279 mg, $0.122 \mathrm{mmol}$ ), PyBOP (507 mg, 8 equiv) and $Z$-D-Ala triethylammonium salt $(217 \mathrm{mg}$, 8 equiv) were dried separately $3 \mathrm{~h}$ in a high vacuum. After this time compound $\mathbf{1 4}$ was dissolved in $10 \mathrm{~mL}$ of dry $\mathrm{CH}_{2} \mathrm{Cl}_{2}, Z$-D-Ala triethylammonium salt and PyBOP were added. $N$-Methylimidazol $(155 \mu \mathrm{L}$, 16 equiv) was added dropwise and the reaction mixture stirred for 2.5-3 h at $\mathrm{rt}$ under an argon atmosphere. The reaction mixture was diluted with $\mathrm{CH}_{2} \mathrm{Cl}_{2}$ and washed with satd $\mathrm{NH}_{4} \mathrm{Cl}$ solution. The organic phase was dried over $\mathrm{MgSO}_{4}$ and the solvent was removed under diminished pressure. Purification by flash chromatography (3:1 toluene-acetone) and (second column: 3:1 toluene-acetone) and (second column: 3:1 toluene-acetone) yielded diastereomer $15(315 \mathrm{mg}, 92 \%)$ as a colorless syrup, which was stored at $-20^{\circ} \mathrm{C}$. TLC (3:1 tolueneacetone): $R_{\mathrm{f}} 0.25, R_{\mathrm{f}} 0.33$. $[\alpha]_{\mathrm{D}}-8.1\left(c 0.8, \mathrm{CHCl}_{3}\right) .{ }^{1} \mathrm{H}$ NMR $\left(600 \mathrm{MHz}, \mathrm{CDCl}_{3}\right): \delta 0.79-0.94(\mathrm{t}, 6 \mathrm{H}, \mathrm{Me})$, 1.09-1.38 (m, 40H, $\mathrm{CH}_{2}$-chain), 1.46-1.61 (m, 4H, $\mathrm{COCH}_{2} \mathrm{CH}_{2} \mathrm{R}$ ), 2.12-2.27 (m, 4H, $\left.\mathrm{COCH}_{2} \mathrm{CH}_{2} \mathrm{R}\right), 3.34$ $(\mathrm{m}, 1 \mathrm{H}, 2 \mathrm{a}-\mathrm{H}), 3.35(\mathrm{~m}, 1 \mathrm{H}, 5 \mathrm{a} / \mathrm{b}-\mathrm{H}), 3,37(\mathrm{~m}, 2 \mathrm{H}, 5 \mathrm{a} /$ b-H, 1'-H), 3.39 (m, 1H, 2b-H), 3.46 (m, 2H, 4a-H, 4b-H), 3.51 (m, 2H, 9-H), $3.58(\mathrm{~m}, 2 \mathrm{H}, 3 \mathrm{a}-\mathrm{H}, 3 \mathrm{~b}-\mathrm{H})$, $3.60(\mathrm{~m}, 1 \mathrm{H}, 6 \mathrm{a}-\mathrm{H}), 3.71(\mathrm{~m}, 1 \mathrm{H}, 8-\mathrm{H}), 3.84(\mathrm{~m}, 1 \mathrm{H}$, $\left.1^{\prime}-\mathrm{H}\right), 4.00$ (m, 8H, 1-H, 3-H, 4-H, 6-H), 4.06 (m, 1H, 7-H), 4.07 (m, 1H, 3'-H), $4.12(\mathrm{~m}, 1 \mathrm{H}, 6 \mathrm{a}-\mathrm{H}), 4.14$ $\left(\mathrm{m}, 1 \mathrm{H}, 3^{\prime}-\mathrm{H}\right), 4.17(\mathrm{~m}, 1 \mathrm{H}, 7-\mathrm{H}), 4.22(\mathrm{~m}, 1 \mathrm{H}, 1 \mathrm{a}-\mathrm{H})$, $4.23(\mathrm{~m}, 1 \mathrm{H}, 6 \mathrm{~b}-\mathrm{H}), 4.32(\mathrm{~m}, 2 \mathrm{H}, \mathrm{CHNHCbz}), 4.42$ $(\mathrm{m}, 1 \mathrm{H}, 1 \mathrm{~b}-\mathrm{H}), 4.45-4.84\left(\mathrm{~m}, 16 \mathrm{H}, \mathrm{CH}_{2} \mathrm{Ph}\right), 4.97(\mathrm{~m}$, $\left.2 \mathrm{H}, \mathrm{CH}_{2} \mathrm{Cbz}\right), 4.99\left(\mathrm{~m}, 6 \mathrm{H}, \mathrm{POCH} \mathrm{H}_{2} \mathrm{Ph}\right), 5.04(\mathrm{~m}$, 2H, CH $\left.\mathrm{CH}_{2} \mathrm{Cbz}\right), 5.08\left(\mathrm{~m}, 1 \mathrm{H}, 2^{\prime}-\mathrm{H}\right), 5.09(\mathrm{~m}, 2 \mathrm{H}, \mathrm{CH}-$ Ala), 5.58-6.15 (br s, NH), 7.07-7.46 (m, 75H, Ph). ${ }^{13} \mathrm{C} \mathrm{NMR}\left(150.9 \mathrm{MHz}, \mathrm{CDCl}_{3}\right): \delta 14.1$ (2C, $\left.\mathrm{Me}\right)$, 18.03 (2C, Ala-Me), 24.8/24.9 (2C, $\left.\mathrm{COCH}_{2} \mathrm{CH}_{2} \mathrm{R}\right)$, 
22.7/29.7/31.9 (20C, $\mathrm{CH}_{2}$-chain), 34.0, 34.2 (2C, $\left.\mathrm{COCH}_{2} \mathrm{R}\right), 49.7$ (2C, CHNHCbz), 62.8 (1C, C-3'), 65.0 (4C, C-1, C-3, C-4, C-6), 66.5 (1C, C-6b), 66.8 (2C, $\left.\mathrm{CH}_{2} \mathrm{Cbz}\right), 67.3(1 \mathrm{C}, \mathrm{C}-7), 68.0\left(1 \mathrm{C}, \mathrm{C}-1^{\prime}\right), 68.6$ (1C, C-6a), 68.8 (1C, C-6a), 68.9 (1C, C-9), 69.8 (1C, C-2'), $70.0\left(3 \mathrm{C}, \mathrm{POCH}_{2} \mathrm{Ph}\right), 70.8$ (2C, CH-Ala), $72.2-75.5\left(8 \mathrm{C}, \mathrm{CH}_{2} \mathrm{Ph}\right), 73.6(1 \mathrm{C}, \mathrm{C}-5 \mathrm{a} / \mathrm{b}), 75.1$ (1C, C-4a/b), 76.4 (1C, C-8), 76.9 (1C, C-4a/b), 77.0 (1C, C-5a/b), 81.9 (2C, C-2a, C-2b), 84.5 (2C, C-3a, C-3b), 103.6 (1C, C-1a), 103.9 (1C, C-1b), 125.3-138.5 $(\mathrm{C}-\mathrm{Ph}), 155.9$ (2C, COCbz), $172.0 \quad\left(3 \mathrm{C}, \mathrm{CO}_{\mathrm{Ala}}\right)$, 172.9/173.2 (2C, COOR). MALDIMS: $\mathrm{m} / z \quad 2721.3$ $[(\mathrm{M}-\mathrm{H})+\mathrm{Na}]^{+}$.

3.7. (2,3-Di-O-benzyl-sn-glycero)-benzyloxyphosphoryl(2-O-(4-methoxybenzyl)-sn-glycero)-benzyloxyphosphoryl-(2-O-(4-methoxybenzyl)-sn-glycero)-benzyloxyphosphoryl-(2-O-(4-methoxybenzyl)-sn-glycero)-benzyloxyphosphoryl-6- $O$-(2,3,4-tri- $O$-benzyl- $\beta$-D-glucopyranosyl)6- $O$-(2,3,4-tri- $O$-benzyl- $\beta$-D-glucopyranosyl)-1,2-di- $O$ myristoyl-sn-glycerol (16)

Compound 16 was synthesized following the procedure described for compound 13. Compound $\mathbf{9}^{17}$ (200 mg, $0.146 \mathrm{mmol})$, tetrazole $(21.6 \mathrm{mg}, 0.292 \mathrm{mmol})$, compound $7^{15}$ (347 mg, 1.5 equiv) in dry $\mathrm{CH}_{2} \mathrm{Cl}_{2}(7 \mathrm{~mL})$. Oxidation with $t$-BuOOH $(0.48 \mathrm{~mL})$. Purification in flash chromatography (2:1 toluene-acetone) yielded $\mathbf{1 6}$ (354 mg, 84\%) as a colorless oil. TLC (1:1 toluene-acetone): $R_{\mathrm{f}}$ 0.55. $[\alpha]_{\mathrm{D}}+3.8$ (c $\left.1, \mathrm{CHCl}_{3}\right) .{ }^{1} \mathrm{H}$ NMR $\left(600 \mathrm{MHz}, \mathrm{CDCl}_{3}\right): \delta 0.88(\mathrm{t}, 6 \mathrm{H}, \mathrm{Me}), 1.08-1.34(\mathrm{~m}$, $40 \mathrm{H}, \mathrm{CH}_{2}$-chain), $1.46-1.59\left(\mathrm{~m}, 4 \mathrm{H}, \mathrm{COCH}_{2} \mathrm{CH}_{2} \mathrm{R}\right.$ ), 2.17-2.26 (m, 4H, $\left.\mathrm{COCH}_{2} \mathrm{CH}_{2} \mathrm{R}\right), 3.35(\mathrm{~m}, 2 \mathrm{H}, 2 \mathrm{a}-\mathrm{H}$, $5 \mathrm{a} / \mathrm{b}-\mathrm{H}), 3,38\left(\mathrm{~m}, 1 \mathrm{H}, 1^{\prime}-\mathrm{H}\right), 3.39(\mathrm{~m}, 2 \mathrm{H}, 2 \mathrm{~b}-\mathrm{H}, 5 \mathrm{a} / \mathrm{b}-$ H), 3.47-3.49 (m, 2H, 4a-H, 4b-H), $3.52(\mathrm{~m}, 2 \mathrm{H}, 12-$ $\mathrm{H}), 3.60(\mathrm{~m}, 2 \mathrm{H}, 3 \mathrm{a}-\mathrm{H}, 3 \mathrm{~b}-\mathrm{H}), 3.61(\mathrm{~m}, 1 \mathrm{H}, 6 \mathrm{a}-\mathrm{H})$, $3.66(\mathrm{~m}, 3 \mathrm{H}, 2-\mathrm{H}, 5-\mathrm{H}, 8-\mathrm{H}), 3.69(\mathrm{~m}, 9 \mathrm{H}, \mathrm{OMe})$, $3.73(\mathrm{~m}, 1 \mathrm{H}, 11-\mathrm{H}), 3.86\left(\mathrm{~m}, 1 \mathrm{H}, 1^{\prime}-\mathrm{H}\right), 3.97-4.05(\mathrm{~m}$, 12H, 1-H, 3-H, 4-H, 6-H, 7-H, 9-H), 4.08 (m, 2H, 11$\left.\mathrm{H}, 3^{\prime}-\mathrm{H}\right), 4.14(\mathrm{~m}, 1 \mathrm{H}, 6 \mathrm{a}-\mathrm{H}), 4.16\left(\mathrm{~m}, 1 \mathrm{H}, 3^{\prime}-\mathrm{H}\right)$, $4.18(\mathrm{~m}, 2 \mathrm{H}, 11-\mathrm{H}, 6 \mathrm{~b}-\mathrm{H}), 4.23(\mathrm{~m}, 1 \mathrm{H}, 1 \mathrm{a}-\mathrm{H}), 4.27$ $(\mathrm{m}, 1 \mathrm{H}, 6 \mathrm{~b}-\mathrm{H}), 4.43(\mathrm{~m}, 1 \mathrm{H}, 1 \mathrm{~b}-\mathrm{H}), 4.44-4.93(\mathrm{~m}$, $\left.26 \mathrm{H}, \mathrm{CH}_{2} \mathrm{Ph}\right), 4.95-5.04\left(\mathrm{~m}, 8 \mathrm{H}, \mathrm{POCH}_{2} \mathrm{Ph}\right), 5.09$ $\left(\mathrm{m}, 1 \mathrm{H}, 2^{\prime}-\mathrm{H}\right), 6.70-6.83\left(\mathrm{~m}, 6 \mathrm{H}, \mathrm{Ph}_{\mathrm{MPM}}\right), 7.08-7.37$ $(\mathrm{m}, 66 \mathrm{H}, \mathrm{Ph}) .{ }^{13} \mathrm{C}$ NMR $\left(150.9 \mathrm{MHz}, \mathrm{CDCl}_{3}\right): \delta 55.1$ (3C, OMe), $62.9\left(1 \mathrm{C}, \mathrm{C}-3^{\prime}\right), 65.8$ (6C, C-1, C-3, C-4, C-6, C-7, C-9), 66.5 (1C, C-6b), 67.0 (1C, C-11), 68.1 (1C, C-1'), 68.7 (1C, C-6a), 69.2 (1C, C-12), 69.5 (4C, $\left.\mathrm{POCH}{ }_{2} \mathrm{Ph}\right), 69.8\left(1 \mathrm{C}, \mathrm{C}-2^{\prime}\right), 71.5-76.5\left(13 \mathrm{C}, \mathrm{CH}_{2} \mathrm{Ph}\right)$, $73.6(1 \mathrm{C}, \mathrm{C}-5 \mathrm{a} / \mathrm{b}), 75.2(1 \mathrm{C}, \mathrm{C}-4 \mathrm{a} / \mathrm{b}), 75.4(3 \mathrm{C}, \mathrm{C}-2$, C-5, C-8), 76.7 (1C, C-11), 77.1 (1C, C-4a/b), 78.0 (1C, C-5a/b), 81.9 (2C, C-2a, C-2b), 84.4 (2C, C-3a, C-3b), 103.6 (1C, C-1a), 104.0 (1C, C-1b). MALDIMS: $m / z 2916.2[\mathrm{M}+\mathrm{Na}]^{+}$. Anal. Calcd for $\mathrm{C}_{163} \mathrm{H}_{204} \mathrm{O}_{38} \mathrm{P}_{4}$ (2893.3): C, 67.62; H, 7.10. Found: C, 67.38; H, 7.31 .
3.8. (2,3-Di- $O$-benzyl-sn-glycero)-benzyloxyphosphoryl(sn-glycero)-benzyloxyphosphoryl-(sn-glycero)-benzyloxyphosphoryl-(sn-glycero)-benzyloxyphosphoryl-6- $O$ (2,3,4-tri-O-benzyl- $\beta$-D-glucopyranosyl)-6-O-(2,3,4-tri$O$-benzyl- $\beta$-D-glucopyranosyl)-1,2-di- $O$-myristoyl-snglycerol (17)

Compound 17 was synthesized following the same procedure as described for compound 14. Compound 16 (354 mg, $0.122 \mathrm{mmol}$ ); acetonitrile/toluene/water $(60: 3: 4,10 \mathrm{~mL}) ; \mathrm{Ce}\left(\mathrm{NH}_{4}\right)_{2}\left(\mathrm{NO}_{3}\right)_{6}(0.796 \mathrm{~g}, 12$ equiv). After flash chromatography (1:1 toluene-acetone), compound 17 was obtained (271 mg, 87\%). TLC (1:1.5 toluene-acetone): $R_{\mathrm{f}} \quad 0.53, \quad R_{\mathrm{f}} 0.62 . \quad[\alpha]_{\mathrm{D}}+3.2$ (c $\left.0.6, \mathrm{CHCl}_{3}\right) .{ }^{1} \mathrm{H}$ NMR $\left(600 \mathrm{MHz}, \mathrm{CDCl}_{3}\right): \delta 0.78$ $0.94(\mathrm{t}, 6 \mathrm{H}, \mathrm{Me}), 1.08-1.38\left(\mathrm{~m}, 40 \mathrm{H}, \mathrm{CH}_{2}\right.$-chain), $1.43-1.60\left(\mathrm{~m}, 4 \mathrm{H}, \mathrm{COCH}_{2} \mathrm{CH}_{2} \mathrm{R}\right), 2.08-2.26(\mathrm{~m}, 4 \mathrm{H}$, $\left.\mathrm{COCH}_{2} \mathrm{CH}_{2} \mathrm{R}\right), 3.34(\mathrm{~m}, 1 \mathrm{H}, 2 \mathrm{a}-\mathrm{H}), 3.36(\mathrm{~m}, 1 \mathrm{H}, 5 \mathrm{a} /$ b-H), 3,39 (m, 3H, 5a/b, $\left.1^{\prime}-\mathrm{H}, 2 \mathrm{~b}-\mathrm{H}\right), 3.48(\mathrm{~m}, 2 \mathrm{H}$, $4 \mathrm{a}-\mathrm{H}, 4 \mathrm{~b}-\mathrm{H}), 3.52(\mathrm{~m}, 2 \mathrm{H}, 12-\mathrm{H}), 3.59(\mathrm{~m}, 2 \mathrm{H}, 3 \mathrm{a}-\mathrm{H}$, 3b-H), $3.61(\mathrm{~m}, 1 \mathrm{H}, 6 \mathrm{a}-\mathrm{H}), 3.72(\mathrm{~m}, 1 \mathrm{H}, 11-\mathrm{H}), 3.85$ $\left(\mathrm{m}, 1 \mathrm{H}, 1^{\prime}-\mathrm{H}\right), 3.96,4.06\left(\mathrm{~m}, 12 \mathrm{H}, \mathrm{CH}_{2 \mathrm{Glyc}}\right), 4.07(\mathrm{~m}$, $\left.1 \mathrm{H}, 3^{\prime}-\mathrm{H}\right), 4.09(\mathrm{~m}, 1 \mathrm{H}, 10-\mathrm{H}), 4.12(\mathrm{~m}, 1 \mathrm{H}, 6 \mathrm{a}-\mathrm{H})$, $4.16(\mathrm{~m}, 1 \mathrm{H}, 10-\mathrm{H}), 4.17(\mathrm{~m}, 1 \mathrm{H}, 6 \mathrm{~b}-\mathrm{H}), 4.18(\mathrm{~m}, 1 \mathrm{H}$, 11-H), $4.24(\mathrm{~m}, 1 \mathrm{H}, 1 \mathrm{a}-\mathrm{H}), 4.30(\mathrm{~m}, 1 \mathrm{H}, 6 \mathrm{~b}-\mathrm{H}), 4.42$ $(\mathrm{m}, 1 \mathrm{H}, 1 \mathrm{~b}-\mathrm{H}), 4.46-4.95\left(\mathrm{~m}, 16 \mathrm{H}, \mathrm{CH}_{2} \mathrm{Ph}\right), 5.02(\mathrm{~m}$, $\left.8 \mathrm{H}, \mathrm{POCH} \mathrm{H}_{2} \mathrm{Ph}\right), 5.09\left(\mathrm{~m}, 1 \mathrm{H}, 2^{\prime}-\mathrm{H}\right), 7.08-7.44(\mathrm{~m}$, $60 \mathrm{H}, \mathrm{Ph}) .{ }^{13} \mathrm{C}$ NMR $\left(150.9 \mathrm{MHz}, \mathrm{CDCl}_{3}\right): \delta 14.1$ $(2 \mathrm{C}, \mathrm{Me}), 24.8\left(2 \mathrm{C}, \mathrm{COCH}_{2} \mathrm{CH}_{2} \mathrm{R}\right), 22.7 / 29.7 / 31.9$ (20C, $\mathrm{CH}_{2}$-chain), 34.1, $34.2\left(2 \mathrm{C}, \mathrm{COCH}_{2} \mathrm{R}\right), 63.8$ (1C, C-3'), 66.5 (1C, C-6b), 67.2 (1C, C-11), 68.1 (C$\left.1^{\prime}, \mathrm{C}-\mathrm{CH}_{2 \mathrm{Glyc}}\right), 68.6$ (1C, C-6a), 68.9 (1C, C-12), 69.7 (4C, $\left.\mathrm{POCH} \mathrm{H}_{2} \mathrm{Ph}\right), 69.8\left(1 \mathrm{C}, \mathrm{C}-2^{\prime}\right), \quad 72.2-76.0$ (8C, $\left.\mathrm{CH}_{2} \mathrm{Ph}\right), 73.5(1 \mathrm{C}, \mathrm{C}-5 \mathrm{a} / \mathrm{b}), 75.0(1 \mathrm{C}, \mathrm{C}-4 \mathrm{a} / \mathrm{b}), 76.4$ (1C, C-11), 76.9 (1C, C-4a/b), 77.8 (1C, C-5a/b), 81.9 (2C, C-2a, C-2b), 84.4 (2C, C-3a, C-3b), 103.6 (1C, C-1a), 103.9 (1C, C-1b), 127.6-138.5 (C-Ph), 172.9/ 173.3 (2C, COOR). MALDIMS: $\mathrm{m} / \mathrm{z} 2556.2$ $[\mathrm{M}+\mathrm{Na}]^{+}$. Anal. Calcd for $\mathrm{C}_{139} \mathrm{H}_{180} \mathrm{O}_{35} \mathrm{P}_{4}$ (2533.1): C, 65.86; H, 7.16. Found: C, 66.11; H, 6.97.

3.9. (2,3-Di-O-benzyl-sn-glycero)-benzyloxyphosphoryl(2-O-( $N$-benzyloxycarbonyl-D-alanyl)-sn-glycero)-benzyloxyphosphoryl-(2-O-( $N$-benzyloxycarbonyl-D-alanyl)-snglycero)-benzyloxyphosphoryl-(2- $O$ - $(N$-benzyloxycarbonyl-D-alanyl)-sn-glycero)-benzyloxyphosphoryl-6- $O$ (2,3,4-tri- $O$-benzyl- $\beta$-D-glucopyranosyl)-6- $O$-(2,3,4-tri$O$-benzyl- $\beta$-D-glucopyranosyl)-1,2-di- $O$-myristoyl-snglycerol (18)

Compound 18 was synthesized following the same procedure as described for compound 15. Compound 17 (261 mg, $0.103 \mathrm{mmol}$ ), PyBOP (804 mg, 15 equiv) and $Z$-D-Ala triethylammonium salt $(370 \mathrm{mg}$, 15 equiv), $N$-methylimidazol ( $330 \mu \mathrm{L}, 24$ equiv). After purification, compound $\mathbf{1 8}$ was obtained (176 mg, 
$56 \%)$. TLC (1:1.5 toluene-acetone): $R_{\mathrm{f}} 0.77, R_{\mathrm{f}} 0.81$. $[\alpha]_{\mathrm{D}}-8.9 \quad\left(c \quad 0.46, \mathrm{CHCl}_{3}\right) .{ }^{1} \mathrm{H} \quad \mathrm{NMR} \quad(600 \mathrm{MHz}$, $\left.\mathrm{CDCl}_{3}\right): \delta 0.79-0.94(\mathrm{t}, 6 \mathrm{H}, \mathrm{Me}), 1.09-1.38(\mathrm{~m}, 40 \mathrm{H}$, $\mathrm{CH}_{2}$-chain), 1.46-1.61 (m, 4H, $\left.\mathrm{COCH}_{2} \mathrm{CH}_{2} \mathrm{R}\right), 2.12-$ $2.27\left(\mathrm{~m}, 4 \mathrm{H}, \mathrm{COCH}_{2} \mathrm{CH}_{2} \mathrm{R}\right), 3.34(\mathrm{~m}, 1 \mathrm{H}, 2 \mathrm{a}-\mathrm{H})$, $3.35(\mathrm{~m}, 1 \mathrm{H}, 5 \mathrm{a} / \mathrm{b}-\mathrm{H}), 3,37\left(\mathrm{~m}, 2 \mathrm{H}, 5 \mathrm{a} / \mathrm{b}-\mathrm{H}, 1^{\prime}-\mathrm{H}\right)$, $3.39(\mathrm{~m}, 1 \mathrm{H}, 2 \mathrm{~b}-\mathrm{H}), 3.46(\mathrm{~m}, 2 \mathrm{H}, 4 \mathrm{a}-\mathrm{H}, 4 \mathrm{~b}-\mathrm{H}), 3.51$ $(\mathrm{m}, 2 \mathrm{H}, 12-\mathrm{H}), 3.58(\mathrm{~m}, 2 \mathrm{H}, 3 \mathrm{a}-\mathrm{H}, 3 \mathrm{~b}-\mathrm{H}), 3.60(\mathrm{~m}$, $1 \mathrm{H}, 6 \mathrm{a}-\mathrm{H}), 3.71(\mathrm{~m}, 1 \mathrm{H}, 11-\mathrm{H}), 3.84\left(\mathrm{~m}, 1 \mathrm{H}, 1^{\prime}-\mathrm{H}\right)$, $4.00(\mathrm{~m}, 12 \mathrm{H}, 1-\mathrm{H}, 3-\mathrm{H}, 4-\mathrm{H}, 6-\mathrm{H}, 7-\mathrm{H}, 9-\mathrm{H}), 4.06$ $(\mathrm{m}, 1 \mathrm{H}, 10-\mathrm{H}), 4.07\left(\mathrm{~m}, 1 \mathrm{H}, 3^{\prime}-\mathrm{H}\right), 4.12(\mathrm{~m}, 1 \mathrm{H}, 6 \mathrm{a}-$ $\mathrm{H}), 4.14\left(\mathrm{~m}, 1 \mathrm{H}, 3^{\prime}-\mathrm{H}\right), 4.17(\mathrm{~m}, 1 \mathrm{H}, 10-\mathrm{H}), 4.22(\mathrm{~m}$, $1 \mathrm{H}, 1 \mathrm{a}-\mathrm{H}), 4.23(\mathrm{~m}, 1 \mathrm{H}, 6 \mathrm{~b}-\mathrm{H}), 4.32(\mathrm{~m}, 3 \mathrm{H}$, CHNHCbz), $4.42(\mathrm{~m}, 1 \mathrm{H}, 1 \mathrm{~b}-\mathrm{H}), 4.45-4.84(\mathrm{~m}, 16 \mathrm{H}$, $\left.\mathrm{CH}_{2} \mathrm{Ph}\right), 4.97\left(\mathrm{~m}, 3 \mathrm{H}, \mathrm{CH}_{2} \mathrm{Cbz}\right), 4.99(\mathrm{~m}, 8 \mathrm{H}$, $\left.\mathrm{POCH} \mathrm{H}_{2} \mathrm{Ph}\right), 5.04\left(\mathrm{~m}, 3 \mathrm{H}, \mathrm{CH}_{2} \mathrm{Cbz}\right), 5.08\left(\mathrm{~m}, 1 \mathrm{H}, 2^{\prime}-\right.$ $\mathrm{H}), \quad 5.09(\mathrm{~m}, 3 \mathrm{H}, \mathrm{CH}$-Ala $), 5.58-6.15(\mathrm{br} \mathrm{s}, \mathrm{NH})$, 7.07-7.46 (m, $75 \mathrm{H}, \mathrm{Ph}) .{ }^{13} \mathrm{C}$ NMR $(150.9 \mathrm{MHz}$, $\left.\mathrm{CDCl}_{3}\right): \delta 14.1(2 \mathrm{C}, \mathrm{Me}), 18.03$ (3C, Ala-Me), 24.8/ $24.9\left(2 \mathrm{C}, \mathrm{COCH}_{2} \mathrm{CH}_{2} \mathrm{R}\right), 22.7 / 29.7 / 31.9\left(20 \mathrm{C}, \mathrm{CH}_{2^{-}}\right.$ chain), 34.0, $34.2 \quad\left(2 \mathrm{C}, \quad \mathrm{COCH}_{2} \mathrm{R}\right), \quad 49.7 \quad(3 \mathrm{C}$, CHNHCbz), 62.8 (1C, C-3'), 65.0 (6C, C-1, C-3, C-4, C-6, C-7, C-9), 66.5 (1C, C-6b), 66.8 (3C, $\left.\mathrm{CH}_{2} \mathrm{Cbz}\right)$, 67.3 (1C, C-10), 68.0 (1C, C-1'), 68.6 (1C, C-6a), 68.8 (1C, C-6a), 68.9 (1C, C-12), 69.8 (1C, C-2'), 70.0 (4C, $\left.\mathrm{POCH}_{2} \mathrm{Ph}\right), \quad 70.8 \quad(3 \mathrm{C}, \mathrm{CH}-\mathrm{Ala}), \quad 72.2-75.5 \quad(8 \mathrm{C}$, $\left.\mathrm{CH}_{2} \mathrm{Ph}\right), 73.6(1 \mathrm{C}, \mathrm{C}-5 \mathrm{a} / \mathrm{b}), 75.1(1 \mathrm{C}, \mathrm{C}-4 \mathrm{a} / \mathrm{b}), 76.4$ (1C, C-11), 76.9 (1C, C-4a/b), 77.0 (1C, C-5a/b), 81.9 (2C, C-2a, C-2b), 84.5 (2C, C-3a, C-3b), 103.6 (1C, C-1a), 103.9 (1C, C-1b), 125.3-138.5 (C-Ph), 155.9 (3C, COCbz), $172.0\left(3 \mathrm{C}, \mathrm{CO}_{\mathrm{Ala}}\right), 172.9 / 173.2(2 \mathrm{C}$, COOR). MALDIMS (positive Mode, Matrix $p$-Nitroaniline + NaI, THF): $[(\mathrm{M}-\mathrm{H})+2 \mathrm{Na}]^{+}, \mathrm{m} / z$ 3148.4; found: $m / z$ 3148.4.

3.10. (2,3-Di-O-benzyl-sn-glycero)-benzyloxyphosphoryl(2-O-(4-methoxybenzyl)-sn-glycero)-benzyloxyphosphoryl-(2-O-(4-methoxybenzyl)-sn-glycero)-benzyloxyphosphoryl-(2-O-(4-methoxybenzyl)-sn-glycero)-benzyloxyphosphoryl-(2-O-(4-methoxybenzyl)-sn-glycero)-benzyloxyphosphoryl-6- $O$-(2,3,4-tri- $O$-benzyl- $\beta$-D-glucopyranosyl)-6- $O$-(2,3,4-tri- $O$-benzyl- $\beta$-D-glucopyranosyl)-1,2-di$O$-myristoyl-sn-glycerol (19)

Compound 19 was synthesized following the procedure described for compound 13. Compound $\mathbf{1 0}^{17}$ (200 mg, $0.116 \mathrm{mmol})$, tetrazole $(24.3 \mathrm{mg}, 0.35 \mathrm{mmol})$, compound $7^{15}$ (280 mg, 1.5 equiv) in dry $\mathrm{CH}_{2} \mathrm{Cl}_{2}$ $(5.5 \mathrm{~mL})$. Oxidation with $t$-BuOOH $(0.38 \mathrm{~mL})$. Purification in flash chromatography (2:1 toluene-acetone) yielded $19(275 \mathrm{mg}, 74 \%)$ as a colorless oil. TLC $(1: 1$ toluene-acetone): $R_{\mathrm{f}} 0.55 .[\alpha]_{\mathrm{D}}+3.6\left(c 1, \mathrm{CHCl}_{3}\right) .{ }^{1} \mathrm{H}$ NMR $\left(600 \mathrm{MHz}, \mathrm{CDCl}_{3}\right): \delta 0.88(\mathrm{t}, 6 \mathrm{H}, \mathrm{Me}), 1.08-$ $1.34\left(\mathrm{~m}, \quad 40 \mathrm{H}, \quad \mathrm{CH}_{2}\right.$-chain), $1.46-1.59 \quad(\mathrm{~m}, \quad 4 \mathrm{H}$, $\left.\mathrm{COCH}_{2} \mathrm{CH}_{2} \mathrm{R}\right), \quad 2.17-2.26\left(\mathrm{~m}, 4 \mathrm{H}, \mathrm{COCH}_{2} \mathrm{CH}_{2} \mathrm{R}\right)$, $3.35(\mathrm{~m}, 2 \mathrm{H}, 2 \mathrm{a}-\mathrm{H}, 5 \mathrm{a} / \mathrm{b}-\mathrm{H}), 3,38\left(\mathrm{~m}, 1 \mathrm{H}, 1^{\prime}-\mathrm{H}\right), 3.39$ (m, 2H, 2b-H, 5a/b-H), 3.47-3.49 (m, 2H, 4a-H, 4b$\mathrm{H}), 3.52(\mathrm{~m}, 2 \mathrm{H}, 15-\mathrm{H}), 3.60(\mathrm{~m}, 2 \mathrm{H}, 3 \mathrm{a}-\mathrm{H}, 3 \mathrm{~b}-\mathrm{H})$, $3.61(\mathrm{~m}, 1 \mathrm{H}, 6 \mathrm{a}-\mathrm{H}), 3.66(\mathrm{~m}, 4 \mathrm{H}, 2-\mathrm{H}, 5-\mathrm{H}, 8-\mathrm{H}, 11-$ $\mathrm{H}), 3.69(\mathrm{~m}, 12 \mathrm{H}, \mathrm{OMe}), 3.73(\mathrm{~m}, 1 \mathrm{H}, 14-\mathrm{H}), 3.86$ $\left(\mathrm{m}, 1 \mathrm{H}, 1^{\prime}-\mathrm{H}\right), 3.97-4.05(\mathrm{~m}, 16 \mathrm{H}, 1-\mathrm{H}, 3-\mathrm{H}, 4-\mathrm{H}, 6-$ $\mathrm{H}, 7-\mathrm{H}, 9-\mathrm{H}, 10-\mathrm{H}, 12-\mathrm{H}), 4.08\left(\mathrm{~m}, 2 \mathrm{H}, 13-\mathrm{H}, 3^{\prime}-\mathrm{H}\right)$, $4.14(\mathrm{~m}, 1 \mathrm{H}, 6 \mathrm{a}-\mathrm{H}), 4.16\left(\mathrm{~m}, 1 \mathrm{H}, 3^{\prime}-\mathrm{H}\right), 4.18(\mathrm{~m}, 2 \mathrm{H}$, 16- $\mathrm{H}, 6 \mathrm{~b}-\mathrm{H}), 4.23(\mathrm{~m}, 1 \mathrm{H}, 1 \mathrm{a}-\mathrm{H}), 4.27(\mathrm{~m}, 1 \mathrm{H}, 6 \mathrm{~b}-$ $\mathrm{H}), 4.43(\mathrm{~m}, 1 \mathrm{H}, 1 \mathrm{~b}-\mathrm{H}), 4.44-4.93\left(\mathrm{~m}, 24 \mathrm{H}, \mathrm{CH}_{2} \mathrm{Ph}\right)$, 4.95-5.04 (m, $\left.10 \mathrm{H}, \mathrm{POCH}_{2} \mathrm{Ph}\right), 5.09\left(\mathrm{~m}, 1 \mathrm{H}, 2^{\prime}-\mathrm{H}\right)$, 6.70-6.83 (m, 8H, $\left.\mathrm{Ph}_{\mathrm{MPM}}\right), 7.08-7.37$ (m, 75H, $\left.\mathrm{Ph}\right)$. ${ }^{13} \mathrm{C}$ NMR $\left(150.9 \mathrm{MHz}, \mathrm{CDCl}_{3}\right): \delta 55.1$ (4C, OMe), $62.9\left(1 \mathrm{C}, \mathrm{C}-3^{\prime}\right), 65.8$ (8C, C-1, C-3, C-4, C-6, C-7, C9, C-10, C-12), 66.5 (1C, C-6b), 67.0 (1C, C-13), 68.1 (1C, C-1'), 68.7 (1C, C-6a), 69.2 (1C, c-18), 69.5 (5C, $\left.\mathrm{POCH}_{2} \mathrm{Ph}\right), 69.8\left(1 \mathrm{C}, \mathrm{C}-2^{\prime}\right), 71.5-76.5\left(12 \mathrm{C}, \mathrm{CH}_{2} \mathrm{Ph}\right)$, $73.6(1 \mathrm{C}, \mathrm{C}-5 \mathrm{a} / \mathrm{b}), 75.2(1 \mathrm{C}, \mathrm{C}-4 \mathrm{a} / \mathrm{b}), 75.4$ (4C, C-2, C-5, C-8, C-11), 76.7 (1C, C-14), 77.1 (1C, C-4a/b), $78.0(1 \mathrm{C}, \mathrm{C}-5 \mathrm{a} / \mathrm{b}), 81.9$ (2C, C-2a, C-2b), 84.4 (2C, C-3a, C-3b), 103.6 (1C, C-1a), 104.0 (1C, C-1b). MALDIMS: $m / z$ 3257.7 $[\mathrm{M}+\mathrm{Na}]^{+}$. Anal. Calcd for $\mathrm{C}_{181} \mathrm{H}_{225} \mathrm{O}_{44} \mathrm{P}_{5}$ (3259.6): C, 66.69; H, 6.96. Found: $\mathrm{C}$, $66.92 ; \mathrm{H}, 7.17$.

\subsection{1. (2,3-Di-O-benzyl-sn-glycero)-benzyloxyphosphoryl- (sn-glycero)-benzyloxyphosphoryl-(sn-glycero)-benzyl- oxyphosphoryl-(sn-glycero)-benzyloxyphosphoryl-(sn-gly- cero)-benzyloxyphosphoryl-6- $O$ - $(2,3,4-$ tri- $O$-benzyl- $\beta$-D- glucopyranosyl)-6- $O$-(2,3,4-tri- $O$-benzyl- $\beta$-D-glucopyr- anosyl)-1,2-di- $O$-myristoyl-sn-glycerol (20)}

Compound 20 was synthesized following the same procedure as described for compound 14. Compound 19 (269 mg, $0.082 \mathrm{mmol}$ ); acetonitrile/toluene/water (60:3:4, $16 \mathrm{~mL}) ; \mathrm{Ce}\left(\mathrm{NH}_{4}\right)_{2}\left(\mathrm{NO}_{3}\right)_{6}$ (720 mg, 16 equiv). After purification, 20 was obtained $(196 \mathrm{mg}, 85 \%)$. TLC (1:1.5 toluene-acetone): $\quad R_{\mathrm{f}} \quad 0.53, \quad R_{\mathrm{f}} \quad 0.62$. $[\alpha]_{\mathrm{D}}+3.8\left(c 0.6, \mathrm{CHCl}_{3}\right) .{ }^{1} \mathrm{H}$ NMR $\left(600 \mathrm{MHz}, \mathrm{CDCl}_{3}\right)$ : $\delta$ 0.78-0.94 (t, 6H, Me), $1.08-1.38\left(\mathrm{~m}, 40 \mathrm{H}, \mathrm{CH}_{2^{-}}\right.$ chain), $1.43-1.60\left(\mathrm{~m}, 4 \mathrm{H}, \mathrm{COCH}_{2} \mathrm{CH}_{2} \mathrm{R}\right), 2.08-2.26$ $\left(\mathrm{m}, 4 \mathrm{H}, \mathrm{COCH}_{2} \mathrm{CH}_{2} \mathrm{R}\right), 3.34(\mathrm{~m}, 1 \mathrm{H}, 2 \mathrm{a}-\mathrm{H}), 3.36(\mathrm{~m}$, $1 \mathrm{H}, 5 \mathrm{a} / \mathrm{b}-\mathrm{H}), 3,39\left(\mathrm{~m}, 3 \mathrm{H}, 5 \mathrm{a} / \mathrm{b}, 1^{\prime}-\mathrm{H}, 2 \mathrm{~b}-\mathrm{H}\right), 3.48$ $(\mathrm{m}, 2 \mathrm{H}, 4 \mathrm{a}-\mathrm{H}, 4 \mathrm{~b}-\mathrm{H}), 3.52(\mathrm{~m}, 2 \mathrm{H}, 15-\mathrm{H}), 3.59(\mathrm{~m}$, $2 \mathrm{H}, 3 \mathrm{a}-\mathrm{H}, 3 \mathrm{~b}-\mathrm{H}), 3.61(\mathrm{~m}, 1 \mathrm{H}, 6 \mathrm{a}-\mathrm{H}), 3.72(\mathrm{~m}, 1 \mathrm{H}$, $14-\mathrm{H}), 3.85\left(\mathrm{~m}, 1 \mathrm{H}, 1^{\prime}-\mathrm{H}\right), 3.96,4.06(\mathrm{~m}, 16 \mathrm{H}$, $\left.\mathrm{CH}_{2 \mathrm{Glyc}}\right), 4.07\left(\mathrm{~m}, 1 \mathrm{H}, 3^{\prime}-\mathrm{H}\right), 4.12(\mathrm{~m}, 1 \mathrm{H}, 6 \mathrm{a}-\mathrm{H})$, $4.16(\mathrm{~m}, 1 \mathrm{H}, 13-\mathrm{H}), 4.17(\mathrm{~m}, 1 \mathrm{H}, 6 \mathrm{~b}-\mathrm{H}), 4.18(\mathrm{~m}, 1 \mathrm{H}$, 13-H), $4.24(\mathrm{~m}, 1 \mathrm{H}, 1 \mathrm{a}-\mathrm{H}), 4.30(\mathrm{~m}, 1 \mathrm{H}, 6 \mathrm{~b}-\mathrm{H}), 4.42$ $(\mathrm{m}, 1 \mathrm{H}, 1 \mathrm{~b}-\mathrm{H}), 4.46-4.95\left(\mathrm{~m}, 16 \mathrm{H}, \mathrm{CH}_{2} \mathrm{Ph}\right), 5.02(\mathrm{~m}$, $\left.10 \mathrm{H}, \mathrm{POCH}_{2} \mathrm{Ph}\right), 5.09\left(\mathrm{~m}, 1 \mathrm{H}, 2^{\prime}-\mathrm{H}\right), 7.08-7.44(\mathrm{~m}$, $60 \mathrm{H}, \mathrm{Ph}) .{ }^{13} \mathrm{C}$ NMR $\left(150.9 \mathrm{MHz}, \mathrm{CDCl}_{3}\right): \delta 14.1$ $(2 \mathrm{C}, \mathrm{Me}), 24.8\left(2 \mathrm{C}, \mathrm{COCH}_{2} \mathrm{CH}_{2} \mathrm{R}\right), 22.7 / 29.7 / 31.9$ (20C, $\mathrm{CH}_{2}$-chain), 34.1, $34.2\left(2 \mathrm{C}, \mathrm{COCH}_{2} \mathrm{R}\right), 63.8$ (1C, C-3'), 66.5 (1C, C-6b), 67.2 (1C, C-16), 68.1 (C$\left.1^{\prime}, \mathrm{C}-\mathrm{CH}_{2 \mathrm{Glyc}}\right), 68.6$ (1C, C-6a), 68.9 (1C, C-18), 69.7 $\left(5 \mathrm{C}, \quad \mathrm{POCH}_{2} \mathrm{Ph}\right), 69.8 \quad\left(1 \mathrm{C}, \mathrm{C}-2^{\prime}\right), \quad 72.2-76.0 \quad(8 \mathrm{C}$, 
$\left.\mathrm{CH}_{2} \mathrm{Ph}\right), 73.5$ (1C, C-5a/b), 75.0 (1C, C-4a/b), 76.4 (1C, C-14), 76.9 (1C, C-4a/b), 77.8 (1C, C-5a/b), 81.9 (2C, C-2a, C-2b), 84.4 (2C, C-3a, C-3b), 103.6 (1C, C-1a), 103.9 (1C, C-1b), 127.6-138.5 (C-Ph), 172.9/173.3 (2C, COOR). MALDIMS: $m / z 2800.2$ $[\mathrm{M}+\mathrm{Na}]^{+}$. Anal. Calcd for $\mathrm{C}_{149} \mathrm{H}_{193} \mathrm{O}_{40} \mathrm{P}_{5}$ (2779.0): $\mathrm{C}$, 64.4; H, 7.00. Found: C, 64.18; H, 7.23.

3.12. (2,3-Di-O-benzyl-sn-glycero)-benzyloxyphosphoryl(2-O-( $\mathrm{N}$-benzyloxycarbonyl-D-alanyl)-sn-glycero)-benzyloxyphosphoryl-(2- $\mathrm{O}$-( $\mathrm{N}$-benzyloxycarbonyl-D-alanyl)-snglycero)-benzyloxyphosphoryl-(2-O - $(\mathrm{N}$-benzyloxycarbonyl-D-alanyl)-sn-glycero)-benzyloxyphosphoryl-(2-O( $N$-benzyloxycarbonyl-D-alanyl)-sn-glycero)-benzyloxyphosphoryl-6- $O$-(2,3,4-tri- $O$-benzyl- $\beta$-D-glucopyranosyl)6-O-(2,3,4-tri- $O$-benzyl- $\beta$-D-glucopyranosyl)-1,2-di- $O$ myristoyl-sn-glycerol (21)

Compound 21 was synthesized following the same procedure as described for compound $\mathbf{1 5}$. Compound $\mathbf{2 0}$ (196 mg, $0.071 \mathrm{mmol}$ ), PyBOP (734 mg, 20 equiv) and $Z$-D-Ala triethylammonium salt (338 $\mathrm{mg}, 20$ equiv), $N$-methylimidazol (230 $\mu \mathrm{L}, 40$ equiv). Compound 21 (174 mg, 69\%). TLC (1:1.5 toluene-acetone): $R_{\mathrm{f}} 0.77$, $R_{\mathrm{f}}$ 0.81. $[\alpha]_{\mathrm{D}}-10 \quad\left(\begin{array}{ccccc}c & 0.46, & \left.\mathrm{CHCl}_{3}\right) . & { }^{1} \mathrm{H} & \mathrm{NMR}\end{array}\right.$ $\left(600 \mathrm{MHz}, \mathrm{CDCl}_{3}\right): \delta 0.79-0.94(\mathrm{t}, 6 \mathrm{H}, \mathrm{Me}), 1.09$ $1.38\left(\mathrm{~m}, 40 \mathrm{H}, \mathrm{CH}_{2}\right.$-chain $), \quad 1.46-1.61(\mathrm{~m}, \quad 4 \mathrm{H}$, $\left.\mathrm{COCH}_{2} \mathrm{CH}_{2} \mathrm{R}\right), \quad 2.12-2.27$ (m, 4H, $\left.\mathrm{COCH}_{2} \mathrm{CH}_{2} \mathrm{R}\right)$, $3.34(\mathrm{~m}, 1 \mathrm{H}, 2 \mathrm{a}-\mathrm{H}), 3.35(\mathrm{~m}, 1 \mathrm{H}, 5 \mathrm{a} / \mathrm{b}-\mathrm{H}), 3,37(\mathrm{~m}$, $\left.2 \mathrm{H}, 5 \mathrm{a} / \mathrm{b}-\mathrm{H}, 1^{\prime}-\mathrm{H}\right), 3.39(\mathrm{~m}, 1 \mathrm{H}, 2 \mathrm{~b}-\mathrm{H}), 3.46(\mathrm{~m}, 2 \mathrm{H}$, $4 \mathrm{a}-\mathrm{H}, 4 \mathrm{~b}-\mathrm{H}), 3.51(\mathrm{~m}, 2 \mathrm{H}, 15-\mathrm{H}), 3.58(\mathrm{~m}, 2 \mathrm{H}, 3 \mathrm{a}-\mathrm{H}$, $3 \mathrm{~b}-\mathrm{H}), 3.60(\mathrm{~m}, 1 \mathrm{H}, 6 \mathrm{a}-\mathrm{H}), 3.71(\mathrm{~m}, 1 \mathrm{H}, 14-\mathrm{H}), 3.84$ $\left(\mathrm{m}, 1 \mathrm{H}, 1^{\prime}-\mathrm{H}\right), 4.00(\mathrm{~m}, 16 \mathrm{H}, 1-\mathrm{H}, 3-\mathrm{H}, 4-\mathrm{H}, 6-\mathrm{H}, 7-$ $\mathrm{H}, 9-\mathrm{H}, 10-\mathrm{H}, 12-\mathrm{H}), 4.06(\mathrm{~m}, 1 \mathrm{H}, 13-\mathrm{H}), 4.07(\mathrm{~m}$, $\left.1 \mathrm{H}, 3^{\prime}-\mathrm{H}\right), 4.12(\mathrm{~m}, 1 \mathrm{H}, 6 \mathrm{a}-\mathrm{H}), 4.14\left(\mathrm{~m}, 1 \mathrm{H}, 3^{\prime}-\mathrm{H}\right)$, $4.17(\mathrm{~m}, 1 \mathrm{H}, 13-\mathrm{H}), 4.22(\mathrm{~m}, 1 \mathrm{H}, 1 \mathrm{a}-\mathrm{H}), 4.23(\mathrm{~m}, 1 \mathrm{H}$, 6b-H), $4.32(\mathrm{~m}, 4 \mathrm{H}, \mathrm{CHNHCbz}), 4.42(\mathrm{~m}, 1 \mathrm{H}, 1 \mathrm{~b}-\mathrm{H})$, 4.45-4.84 (m, 16H, $\left.\mathrm{CH}_{2} \mathrm{Ph}\right), 4.97\left(\mathrm{~m}, 4 \mathrm{H}, \mathrm{CH}_{2} \mathrm{Cbz}\right)$, $4.99\left(\mathrm{~m}, 10 \mathrm{H}, \mathrm{POCH}_{2} \mathrm{Ph}\right), 5.04\left(\mathrm{~m}, 4 \mathrm{H}, \mathrm{CH}_{2} \mathrm{Cbz}\right)$, $5.08\left(\mathrm{~m}, 1 \mathrm{H}, 2^{\prime}-\mathrm{H}\right), 5.09(\mathrm{~m}, 4 \mathrm{H}, \mathrm{CH}$-Ala), $5.58-6.15$ (br s, NH), 7.07-7.46 (m, 85H, Ph). ${ }^{13} \mathrm{C}$ NMR $\left(150.9 \mathrm{MHz}, \mathrm{CDCl}_{3}\right): \delta 14.1$ (2C, Me), 18.03 (4C, Ala-Me), 24.8/24.9 (2C, $\left.\mathrm{COCH}_{2} \mathrm{CH}_{2} \mathrm{R}\right), \quad 22.7 / 29.7 /$ 31.9 (20C, $\mathrm{CH}_{2}$-chain), 34.0, $34.2\left(2 \mathrm{C}, \mathrm{COCH}_{2} \mathrm{R}\right)$, 49.7 (4C, CHNHCbz), 62.8 (1C, C-3'), 65.0 (8C, C-1, C-3, C-4, C-6, C-7, C-9, C-10, C-12), 66.5 (1C, C6b), 66.8 (4C, $\left.\mathrm{CH}_{2} \mathrm{Cbz}\right), 67.3$ (1C, C-13), 68.0 (1C, C-1'), 68.6 (1C, C-6a), 68.8 (1C, C-6a), 68.9 (1C, C15), $69.8\left(1 \mathrm{C}, \mathrm{C}-2^{\prime}\right), 70.0\left(5 \mathrm{C}, \mathrm{POCH}_{2} \mathrm{Ph}\right), 70.8(4 \mathrm{C}$, CH-Ala), $72.2-75.5\left(8 \mathrm{C}, \mathrm{CH}_{2} \mathrm{Ph}\right), 73.6(1 \mathrm{C}, \mathrm{C}-5 \mathrm{a} / \mathrm{b})$, 75.1 (1C, C-4a/b), 76.4 (1C, C-14), 76.9 (1C, C-4a/b), $77.0(1 \mathrm{C}, \mathrm{C}-5 \mathrm{a} / \mathrm{b}), 81.9$ (2C, C-2a, C-2b), 84.5 (2C, C-3a, C-3b), 103.6 (1C, C-1a), 103.9 (1C, C-1b), 125.3-138.5 (C-Ph), 155.9 (4C, COCbz), 172.0 (4C, $\left.\mathrm{CO}_{\mathrm{Ala}}\right), 172.9 / 173.2$ (2C, COOR). MALDIMS: $\mathrm{m} / \mathrm{z}$ $3642.3[(\mathrm{M}-\mathrm{H})+2 \mathrm{Na}]^{+}$.
3.13. (2,3-Di-O-benzyl-sn-glycero)-benzyloxyphosphoryl(2-O-(4-methoxybenzyl)-sn-glycero)-benzyloxyphosphoryl-(2-O-(4-methoxybenzyl)-sn-glycero)-benzyloxyphosphoryl-(2-O-(4-methoxybenzyl)-sn-glycero)-benzyloxyphosphoryl-(2-O-(4-methoxybenzyl)-sn-glycero)-benzyloxyphosphoryl-(2-O-(4-methoxybenzyl)-sn-glycero)-benzyloxyphosphoryl-6- $O-(2,3,4$-tri- $O$-benzyl- $\beta$-D-glucopyranosyl)-6- $O$-(2,3,4-tri- $O$-benzyl- $\beta$-D-glucopyranosyl)-1,2di- $O$-myristoyl-sn-glycerol (22)

Compound 22 was synthesized following the procedure described for compound 13. Compound 12 (870 mg, $0.416 \mathrm{mmol}$ ), tetrazole (58 $\mathrm{mg}, 2$ equiv), compound $7^{15}$ (818 mg, 1.3 equiv) in dry $\mathrm{CH}_{2} \mathrm{Cl}_{2}(15 \mathrm{~mL})$. Oxidation with $t$-BuOOH $(1.2 \mathrm{~mL})$. Purification in flash chromatography (2:1 toluene-acetone) yielded $22(1 \mathrm{~g}, 67 \%)$ as a colorless oil. TLC (1:1 toluene-acetone): $R_{\mathrm{f}} 0.55$. $[\alpha]_{\mathrm{D}}+3.3\left(c 1, \mathrm{CHCl}_{3}\right) .{ }^{1} \mathrm{H}$ NMR $\left(600 \mathrm{MHz}, \mathrm{CDCl}_{3}\right)$ : $\delta 0.88(\mathrm{t}, 6 \mathrm{H}, \mathrm{Me}), 1.08-1.34\left(\mathrm{~m}, 40 \mathrm{H}, \mathrm{CH}_{2}\right.$-chain), 1.46-1.59 (m, 4H, $\left.\mathrm{COCH}_{2} \mathrm{CH}_{2} \mathrm{R}\right), 2.17-2.26(\mathrm{~m}, 4 \mathrm{H}$, $\left.\mathrm{COCH}_{2} \mathrm{CH}_{2} \mathrm{R}\right), 3.35(\mathrm{~m}, 2 \mathrm{H}, 2 \mathrm{a}-\mathrm{H}, 5 \mathrm{a} / \mathrm{b}-\mathrm{H}), 3,38(\mathrm{~m}$, $\left.1 \mathrm{H}, 1^{\prime}-\mathrm{H}\right), 3.39(\mathrm{~m}, 2 \mathrm{H}, 2 \mathrm{~b}-\mathrm{H}, 5 \mathrm{a} / \mathrm{b}-\mathrm{H}), 3.47-3.49(\mathrm{~m}$, $2 \mathrm{H}, 4 \mathrm{a}-\mathrm{H}, 4 \mathrm{~b}-\mathrm{H}), 3.52(\mathrm{~m}, 2 \mathrm{H}, 18-\mathrm{H}), 3.60(\mathrm{~m}, 2 \mathrm{H}, 3 \mathrm{a}-$ $\mathrm{H}, 3 \mathrm{~b}-\mathrm{H}), 3.61(\mathrm{~m}, 1 \mathrm{H}, 6 \mathrm{a}-\mathrm{H}), 3.66(\mathrm{~m}, 5 \mathrm{H}, 2-\mathrm{H}, 5-\mathrm{H}$, 8-H, 11-H, 14-H), $3.69(\mathrm{~m}, 15 \mathrm{H}, \mathrm{OMe}), 3.73(\mathrm{~m}, 1 \mathrm{H}$, $17-\mathrm{H}), 3.86\left(\mathrm{~m}, 1 \mathrm{H}, 1^{\prime}-\mathrm{H}\right), 3.97-4.05(\mathrm{~m}, 20 \mathrm{H}, 1-\mathrm{H}, 3-$ $\mathrm{H}, 4-\mathrm{H}, 6-\mathrm{H}, 7-\mathrm{H}, 9-\mathrm{H}, 10-\mathrm{H}, 12-\mathrm{H}, 13-\mathrm{H}, 15-\mathrm{H}), 4.08$ $\left(\mathrm{m}, 2 \mathrm{H}, 16-\mathrm{H}, 3^{\prime}-\mathrm{H}\right), 4.14(\mathrm{~m}, 1 \mathrm{H}, 6 \mathrm{a}-\mathrm{H}), 4.16(\mathrm{~m}, 1 \mathrm{H}$, $\left.3^{\prime}-\mathrm{H}\right), 4.18(\mathrm{~m}, 2 \mathrm{H}, 16-\mathrm{H}, 6 \mathrm{~b}-\mathrm{H}), 4.23(\mathrm{~m}, 1 \mathrm{H}, 1 \mathrm{a}-\mathrm{H})$, $4.27(\mathrm{~m}, 1 \mathrm{H}, 6 \mathrm{~b}-\mathrm{H}), 4.43(\mathrm{~m}, 1 \mathrm{H}, 1 \mathrm{~b}-\mathrm{H}), 4.44-4.93(\mathrm{~m}$, $\left.26 \mathrm{H}, \mathrm{CH}_{2} \mathrm{Ph}\right), 4.95-5.04\left(\mathrm{~m}, 12 \mathrm{H}, \mathrm{POCH}_{2} \mathrm{Ph}\right), 5.09(\mathrm{~m}$, $\left.1 \mathrm{H}, 2^{\prime}-\mathrm{H}\right), 6.70-6.83\left(\mathrm{~m}, 10 \mathrm{H}, \mathrm{Ph}_{\mathrm{MPM}}\right), 7.08-7.37(\mathrm{~m}$, $80 \mathrm{H}, \mathrm{Ph}) .{ }^{13} \mathrm{C}$ NMR $\left(150.9 \mathrm{MHz}, \mathrm{CDCl}_{3}\right): \delta 55.1(5 \mathrm{C}$, OMe), 62.9 (1C, C-3'), 65.8 (10 C, C-1, C-3, C-4, C-6, C-7, C-9, C-10, C-12, C-13, C-15), 66.5 (1C, C-6b), 67.0 (1C, C-16), 68.1 (1C, C-1'), 68.7 (1C, C-6a), 69.2 (1C, c-18), $69.5\left(6 \mathrm{C}, \mathrm{POCH}_{2} \mathrm{Ph}\right), 69.8\left(1 \mathrm{C}, \mathrm{C}-2^{\prime}\right), 71.5$ $76.5\left(13 \mathrm{C}, \mathrm{CH}_{2} \mathrm{Ph}\right), 73.6(1 \mathrm{C}, \mathrm{C}-5 \mathrm{a} / \mathrm{b}), 75.2(1 \mathrm{C}, \mathrm{C}-4 \mathrm{a} /$ b), 75.4 (5C, C-2, C-5, C-8, C-11, C-14), 76.7 (1C, C17), $77.1(1 \mathrm{C}, \mathrm{C}-4 \mathrm{a} / \mathrm{b}), 78.0(1 \mathrm{C}, \mathrm{C}-5 \mathrm{a} / \mathrm{b}), 81.9$ (2C, C2a, C-2b), 84.4 (2C, C-3a, C-3b), 103.6 (1C, C-1a), 104.0 (1C, C-1b). MALDIMS: $m / z$ 3647.7 [M+Na $]^{+}$. Anal. Calcd for $\mathrm{C}_{199} \mathrm{H}_{247} \mathrm{O}_{50} \mathrm{P}_{6}$ (3624.9): C, 65.94; H, 6.87. Found: C, 65.78; H, 7.04.

3.14. (2,3-Di-O-benzyl-sn-glycero)-benzyloxyphosphoryl(sn-glycero)-benzyloxyphosphoryl-(sn-glycero)-benzyloxyphosphoryl-(sn-glycero)-benzyloxyphosphoryl-(sn-glycero)-benzyloxyphosphoryl-(sn-glycero)-benzyloxyphosphoryl-6- $O$-(2,3,4-tri- $O$-benzyl- $\beta$-D-glucopyranosyl)-6- $O$ (2,3,4-tri- $O$-benzyl- $\beta$-D-glucopyranosyl)-1,2-di- $O$-myristoyl-sn-glycerol (23)

Compound 23 was synthesized following the same procedure as described for compound 14. Compound 22 (810 mg, $0.224 \mathrm{mmol}$ ); acetonitrile/toluene/water (60: 
3:4, $37 \mathrm{~mL}) ; \mathrm{Ce}\left(\mathrm{NH}_{4}\right)_{2}\left(\mathrm{NO}_{3}\right)_{6}(3.07 \mathrm{~g}, 25$ equiv). After purification $(1: 1 \rightarrow 1: 3$ toluene-acetone), $\mathbf{2 3}$ was obtained (538 mg, 80\%). TLC (1:1.5 toluene-acetone): $R_{\mathrm{f}} 0.53, R_{\mathrm{f}}$ 0.62. $[\alpha]_{\mathrm{D}}+4.2\left(c 0.6, \mathrm{CHCl}_{3}\right) .{ }^{1} \mathrm{H}$ NMR $(600 \mathrm{MHz}$, $\left.\mathrm{CDCl}_{3}\right): \delta 0.78-0.94(\mathrm{t}, 6 \mathrm{H}, \mathrm{Me}), 1.08-1.38(\mathrm{~m}, 40 \mathrm{H}$, $\mathrm{CH}_{2}$-chain), $1.43-1.60\left(\mathrm{~m}, 4 \mathrm{H}, \mathrm{COCH}_{2} \mathrm{CH}_{2} \mathrm{R}\right), 2.08-$ $2.26\left(\mathrm{~m}, 4 \mathrm{H}, \mathrm{COCH}_{2} \mathrm{CH}_{2} \mathrm{R}\right), 3.34(\mathrm{~m}, 1 \mathrm{H}, 2 \mathrm{a}-\mathrm{H}), 3.36$ (m, 1H, 5a/b-H), 3,39 (m, 3H, 5a/b, 1'-H, 2b-H), 3.48 $(\mathrm{m}, 2 \mathrm{H}, 4 \mathrm{a}-\mathrm{H}, 4 \mathrm{~b}-\mathrm{H}), 3.52(\mathrm{~m}, 2 \mathrm{H}, 18-\mathrm{H}), 3.59(\mathrm{~m}, 2 \mathrm{H}$, $3 \mathrm{a}-\mathrm{H}, 3 \mathrm{~b}-\mathrm{H}), 3.61(\mathrm{~m}, 1 \mathrm{H}, 6 \mathrm{a}-\mathrm{H}), 3.72(\mathrm{~m}, 1 \mathrm{H}, 17-\mathrm{H})$, $3.85\left(\mathrm{~m}, 1 \mathrm{H}, 1^{\prime}-\mathrm{H}\right), 3.96,4.06\left(\mathrm{~m}, 20 \mathrm{H}, \mathrm{CH}_{2 \mathrm{Glyc}}\right), 4.07$ (m, 1H, 3'-H), $4.09(\mathrm{~m}, 1 \mathrm{H}, 16-\mathrm{H}), 4.12(\mathrm{~m}, 1 \mathrm{H}, 6 \mathrm{a}-\mathrm{H})$, $4.16(\mathrm{~m}, 1 \mathrm{H}, 16-\mathrm{H}), 4.17(\mathrm{~m}, 1 \mathrm{H}, 6 \mathrm{~b}-\mathrm{H}), 4.18(\mathrm{~m}, 1 \mathrm{H}$, $16-\mathrm{H}), 4.24(\mathrm{~m}, 1 \mathrm{H}, 1 \mathrm{a}-\mathrm{H}), 4.30(\mathrm{~m}, 1 \mathrm{H}, 6 \mathrm{~b}-\mathrm{H}), 4.42(\mathrm{~m}$, $1 \mathrm{H}, 1 \mathrm{~b}-\mathrm{H}), 4.46-4.95\left(\mathrm{~m}, 16 \mathrm{H}, \mathrm{CH}_{2} \mathrm{Ph}\right), 5.02(\mathrm{~m}, 12 \mathrm{H}$, $\left.\mathrm{POCH} \mathrm{H}_{2} \mathrm{Ph}\right), 5.09\left(\mathrm{~m}, 1 \mathrm{H}, 2^{\prime}-\mathrm{H}\right), 7.08-7.44(\mathrm{~m}, 70 \mathrm{H}, \mathrm{Ph})$. ${ }^{13} \mathrm{C}$ NMR $\left(150.9 \mathrm{MHz}, \mathrm{CDCl}_{3}\right): \delta 14.1(2 \mathrm{C}, \mathrm{Me}), 24.8$ (2C, $\left.\mathrm{COCH}_{2} \mathrm{CH}_{2} \mathrm{R}\right), 22.7 / 29.7 / 31.9$ (20C, $\mathrm{CH}_{2}$-chain), $34.1,34.2\left(2 \mathrm{C}, \mathrm{COCH}_{2} \mathrm{R}\right), 63.8\left(1 \mathrm{C}, \mathrm{C}-3^{\prime}\right), 66.5(1 \mathrm{C}, \mathrm{C}-$ 6b), 67.2 (1C, C-16), 68.1 (C-1', C-CH $\left.\mathrm{CH}_{2 \mathrm{Glyc}}\right), 68.6$ (1C, C-6a), 68.9 (1C, C-18), 69.7 (6C, $\left.\mathrm{POCH}_{2} \mathrm{Ph}\right), 69.8$ (1C, C-2'), 72.2-76.0 (8C, $\left.\mathrm{CH}_{2} \mathrm{Ph}\right), 73.5(1 \mathrm{C}, \mathrm{C}-5 \mathrm{a} / \mathrm{b}), 75.0$ (1C, C-4a/b), 76.4 (1C, C-17), 76.9 (1C, C-4a/b), 77.8 (1C, C-5a/b), 81.9 (2C, C-2a, C-2b), 84.4 (2C, C-3a, C3b), 103.6 (1C, C-1a), 103.9 (1C, C-1b), 127.6-138.5 (C-Ph), 172.9/173.3 (2C, COOR). MALDIMS: $\mathrm{m} / \mathrm{z}$ $3047.6[\mathrm{M}+\mathrm{Na}]^{+}$. Anal. Calcd for $\mathrm{C}_{159} \mathrm{H}_{206} \mathrm{O}_{45} \mathrm{P}_{6}$ (3023.2): C, 63.17; H, 6.87. Found: C, 63.21; H, 7.17.

3.15. (2,3-Di- $O$-benzyl-sn-glycero)-benzyloxyphosphoryl(2-O-( $\mathrm{N}$-benzyloxycarbonyl-D-alanyl)-sn-glycero)-benzyloxyphosphoryl-(2-O-( $\mathrm{N}$-benzyloxycarbonyl-D-alanyl)-snglycero)-benzyloxyphosphoryl-(2-O- $(\mathrm{N}$-benzyloxycarbonyl-D-alanyl)-sn-glycero)-benzyloxyphosphoryl-(2-O( $N$-benzyloxycarbonyl-D-alanyl)-sn-glycero)-benzyloxyphosphoryl-(2-O-( $\mathrm{N}$-benzyloxycarbonyl-D-alanyl)-sn-glycero)-benzyloxyphosphoryl-6- $O$ - $(2,3,4$-tri- $O$-benzyl- $\beta$-Dglucopyranosyl)-6- $O$-(2,3,4-tri- $O$-benzyl- $\beta$-D-glucopyranosyl)-1,2-di- $O$-myristoyl-sn-glycerol (24)

Compound 24 was synthesized following the same procedure as described for compound 15. Compound $\mathbf{2 3}$ ( $310 \mathrm{mg}, 0.103 \mathrm{mmol}$ ), PyBOP ( $1.34 \mathrm{~g}, 25$ equiv) and $Z$ D-Ala triethylammonium salt $(839 \mathrm{mg}, 25$ equiv), $N$ methylimidazol ( $414 \mu \mathrm{L}, 40$ equiv). Compound $\mathbf{2 4}$ (240 mg, 58\%). TLC (1:1.5 toluene-acetone): $R_{\mathrm{f}} 0.77$, $R_{\mathrm{f}} 0.81 .[\alpha]_{\mathrm{D}}-11\left(c 0.46, \mathrm{CHCl}_{3}\right) .{ }^{1} \mathrm{H}$ NMR $(600 \mathrm{MHz}$, $\left.\mathrm{CDCl}_{3}\right): \delta 0.79-0.94(\mathrm{t}, 6 \mathrm{H}, \mathrm{Me}), 1.09-1.38(\mathrm{~m}, 40 \mathrm{H}$, $\mathrm{CH}_{2}$-chain), 1.46-1.61 (m, 4H, $\left.\mathrm{COCH}_{2} \mathrm{CH}_{2} \mathrm{R}\right), 2.12-$ $2.27\left(\mathrm{~m}, 4 \mathrm{H}, \mathrm{COCH}_{2} \mathrm{CH}_{2} \mathrm{R}\right), 3.34(\mathrm{~m}, 1 \mathrm{H}, 2 \mathrm{a}-\mathrm{H}), 3.35$ $(\mathrm{m}, 1 \mathrm{H}, 5 \mathrm{a} / \mathrm{b}-\mathrm{H}), 3,37\left(\mathrm{~m}, 2 \mathrm{H}, 5 \mathrm{a} / \mathrm{b}-\mathrm{H}, 1^{\prime}-\mathrm{H}\right), 3.39(\mathrm{~m}$, $1 \mathrm{H}, 2 \mathrm{~b}-\mathrm{H}), 3.46(\mathrm{~m}, 2 \mathrm{H}, 4 \mathrm{a}-\mathrm{H}, 4 \mathrm{~b}-\mathrm{H}), 3.51(\mathrm{~m}, 2 \mathrm{H}, 18-$ $\mathrm{H}), 3.58(\mathrm{~m}, 2 \mathrm{H}, 3 \mathrm{a}-\mathrm{H}, 3 \mathrm{~b}-\mathrm{H}), 3.60(\mathrm{~m}, 1 \mathrm{H}, 6 \mathrm{a}-\mathrm{H}), 3.71$ $(\mathrm{m}, 1 \mathrm{H}, 17-\mathrm{H}), 3.84\left(\mathrm{~m}, 1 \mathrm{H}, 1^{\prime}-\mathrm{H}\right), 4.00(\mathrm{~m}, 20 \mathrm{H}, 1-\mathrm{H}$, 3-H, 4-H, 6-H, 7-H, 9-H, 10-H, 12-H, 13-H, 15-H), $4.06(\mathrm{~m}, 1 \mathrm{H}, 16-\mathrm{H}), 4.07\left(\mathrm{~m}, 1 \mathrm{H}, 3^{\prime}-\mathrm{H}\right), 4.12(\mathrm{~m}, 1 \mathrm{H}$,
6a-H), $4.14\left(\mathrm{~m}, 1 \mathrm{H}, 3^{\prime}-\mathrm{H}\right), 4.17(\mathrm{~m}, 1 \mathrm{H}, 16-\mathrm{H}), 4.22(\mathrm{~m}$, $1 \mathrm{H}, 1 \mathrm{a}-\mathrm{H}), 4.23(\mathrm{~m}, 1 \mathrm{H}, 6 \mathrm{~b}-\mathrm{H}), 4.32(\mathrm{~m}, 5 \mathrm{H}, \mathrm{CHNHCbz})$, $4.42(\mathrm{~m}, 1 \mathrm{H}, 1 \mathrm{~b}-\mathrm{H}), 4.45-4.84\left(\mathrm{~m}, 16 \mathrm{H}, \mathrm{CH}_{2} \mathrm{Ph}\right), 4.97(\mathrm{~m}$, $\left.5 \mathrm{H}, \mathrm{CH}_{2} \mathrm{Cbz}\right), 4.99\left(\mathrm{~m}, 12 \mathrm{H}, \mathrm{POCH}_{2} \mathrm{Ph}\right), 5.04(\mathrm{~m}, 5 \mathrm{H}$, $\left.\mathrm{CH}_{2} \mathrm{Cbz}\right), 5.08\left(\mathrm{~m}, 1 \mathrm{H}, 2^{\prime}-\mathrm{H}\right), 5.09(\mathrm{~m}, 5 \mathrm{H}, \mathrm{CH}-\mathrm{Ala})$, 5.58-6.15 (br s, NH), 7.07-7.46 (m, 95H, Ph). ${ }^{13} \mathrm{C}$ NMR (150.9 MHz, $\left.\mathrm{CDCl}_{3}\right): \delta 14.1$ (2C, Me), 18.03 (5C, Ala-Me), 24.8/24.9 (2C, $\left.\mathrm{COCH}_{2} \mathrm{CH}_{2} \mathrm{R}\right), 22.7 / 29.7 / 31.9$ (20C, $\mathrm{CH}_{2}$-chain), 34.0, $34.2\left(2 \mathrm{C}, \mathrm{COCH}_{2} \mathrm{R}\right), 49.7$ (5C, CHNHCbz), 62.8 (1C, C-3'), 65.0 (10 C, C-1, C-3, C-4, C-6, C-7, C-9, C-10, C-12, C-13, C-15), 66.5 (1C, C-6b), $66.8\left(5 \mathrm{C}, \mathrm{CH}_{2} \mathrm{Cbz}\right), 67.3$ (1C, C-16), $68.0\left(1 \mathrm{C}, \mathrm{C}-1^{\prime}\right)$, 68.6 (1C, C-6a), 68.8 (1C, C-6a), 68.9 (1C, C-18), 69.8 (1C, C-2'), $70.0\left(6 \mathrm{C}, \mathrm{POCH}_{2} \mathrm{Ph}\right), 70.8$ (5C, CH-Ala), $72.2-75.5\left(8 \mathrm{C}, \mathrm{CH}_{2} \mathrm{Ph}\right), 73.6(1 \mathrm{C}, \mathrm{C}-5 \mathrm{a} / \mathrm{b}), 75.1$ (1C, C4a/b), 76.4 (1C, C-17), 76.9 (1C, C-4a/b), 77.0 (1C, C5a/b), 81.9 (2C, C-2a, C-2b), 84.5 (2C, C-3a, C-3b), 103.6 (1C, C-1a), 103.9 (1C, C-1b), 125.3-138.5 (C-Ph), 155.9 (5C, COCbz), $172.0\left(5 \mathrm{C}, \mathrm{CO}_{\mathrm{Ala}}\right), 172.9 / 173.2(2 \mathrm{C}$, COOR). MALDIMS: $m / z 4069.8[(\mathrm{M}-\mathrm{H})+2 \mathrm{Na}]^{+}$.

3.16. $s n$-Glycero-hydroxyphosphoryl-(2-O-D-alanyl-snglycero)-hydroxyphosphoryl-(2- $O$-D-alanyl-sn-glycero)hydroxyphosphoryl-6- $O$-( $\beta$-D-glucopyranosyl)-6- $O$-( $\beta$-Dglucopyranosyl)-1,2-di- $O$-myristoyl-sn-glycerol (1)

The diastereomers $15(180 \mathrm{mg}, 0.067 \mathrm{mmol})$ were dissolved in $\mathrm{CH}_{2} \mathrm{Cl}_{2} / \mathrm{MeOH} / \mathrm{H}_{2} \mathrm{O}$ (7.5:7.5:1.5, $5 \mathrm{~mL}$ ), treated with Pearlman's catalyst (10\% in weight) and under hydrogen atmosphere, with a $\mathrm{H}_{2}$-filled balloon, was stirred overnight at $\mathrm{rt}$. The reaction was filtrated through Celite, washed with $\mathrm{CH}_{2} \mathrm{Cl}_{2} / \mathrm{MeOH} / \mathrm{H}_{2} \mathrm{O}$ (7.5:7.5:1.5, $4 \mathrm{~mL}$ ) and the filtrate was diluted with $0.1 \mathrm{M}$ $\mathrm{NH}_{4} \mathrm{OAc}-$ buffer ( $\mathrm{pH} 4.8$ ). The solvent was lyophilized and purified using hydrophobic interactions HPLC. After lyophilization, compound $1(29.5 \mathrm{mg}, 32 \%)$ was obtained as white powder. ${ }^{1} \mathrm{H}$ NMR $\left(600 \mathrm{MHz}, \mathrm{D}_{2} \mathrm{O}\right)$ : $\delta$ 0.77-0.93 (m, 6H, Me), 1.12-1.43 (m, 40H, $\mathrm{CH}_{2}-$ chain), 1.51-1.71 (m, 13H, Ala-Me, $\left.\mathrm{COCH}_{2} \mathrm{CH}_{2} \mathrm{R}\right)$, 2.23-2.48 (m, 4H, $\left.\mathrm{COCH}_{2} \mathrm{CH}_{2} \mathrm{R}\right), 3.23-4.62(\mathrm{~m}, 28 \mathrm{H})$, 5.28-5.45 (m, 3H, CH-Ala, $\left.\quad 2^{\prime}-\mathrm{H}\right) .{ }^{13} \mathrm{C} \quad \mathrm{NMR}$ $\left(150.9 \mathrm{MHz}, \mathrm{D}_{2} \mathrm{O}\right): \delta 16.6$ (2C, Me), 18.1 (2C, AlaMe), 24.0 (2C, $\left.\mathrm{COCH}_{2} \mathrm{CH}_{2} \mathrm{R}\right), 25.7 / 33.0 / 35.1$ (20C, $\mathrm{CH}_{2}$-chain), 52.0 (2C, $\left.\mathrm{CHNH}_{3}{ }^{+}\right), 65.1$ (1C, C-9), 66.8 $\left(\mathrm{C}-\mathrm{CH}_{2 \mathrm{Glyc}}\right), 69.8$ (1C, C-7), 73.9 (1C, C-8), 77.0 (3C, $\mathrm{CH}-\mathrm{Ala}, \mathrm{C}-2^{\prime}$ ). MALDIMS (negative mode): $\mathrm{m} / \mathrm{z}$ $1439.6[\mathrm{M}-\mathrm{H}]^{-}, 1369.5[(\mathrm{M}-\mathrm{Ala})-\mathrm{H}]^{-}$.

3.17. $s n$-Glycero-hydroxyphosphoryl-(2-O-D-alanyl-snglycero)-hydroxyphosphoryl-(2- $O$-D-alanyl-sn-glycero)hydroxyphosphoryl-(2-O-D-alanyl-sn-glycero)-hydroxyphosphoryl-6- $O$-( $\beta$-D-glucopyranosyl)-6- $O$-( $\beta$-D-glucopyranosyl)-1,2-di- $O$-myristoyl-sn-glycerol (2)

Compound $\mathbf{2}$ was synthesized following the same procedure as described for compound 1. Compound $\mathbf{1 8}$ 
(174 mg, $\quad 0.021 \mathrm{mmol}) ; \quad \mathrm{CH}_{2} \mathrm{Cl}_{2} / \mathrm{MeOH} / \mathrm{H}_{2} \mathrm{O}$ (7.5:7.5: $1.5,6 \mathrm{~mL})$. Compound $2(38 \mathrm{mg}, 41 \%)$ was obtained as a white powder. ${ }^{1} \mathrm{H}$ NMR $\left(600 \mathrm{MHz}, \mathrm{D}_{2} \mathrm{O}\right): \delta 0.77-$ $0.93(\mathrm{~m}, 6 \mathrm{H}, \mathrm{Me}), 1.12-1.43\left(\mathrm{~m}, 40 \mathrm{H}, \mathrm{CH}_{2}\right.$-chain), 1.51-1.71 (m, 13H, Ala-Me, $\left.\mathrm{COCH}_{2} \mathrm{CH}_{2} \mathrm{R}\right), 2.23-2.48$ (m, $\left.4 \mathrm{H}, \mathrm{COCH}_{2} \mathrm{CH}_{2} \mathrm{R}\right), 3.23-4.62(\mathrm{~m}, 38 \mathrm{H}), 5.28-5.45$ (m, 4H, CH-Ala, $\left.2^{\prime}-\mathrm{H}\right) .{ }^{13} \mathrm{C}$ NMR $\left(150.9 \mathrm{MHz}, \mathrm{D}_{2} \mathrm{O}\right)$ : $\delta 16.6$ (2C, Me), 18.1 (3C, Ala-Me), 24.0 (2C, $\mathrm{COCH}_{2} \mathrm{CH}_{2} \mathrm{R}$ ), 25.7/33.0/35.1 (20C, $\mathrm{CH}_{2}$-chain), 52.0 $\left(3 \mathrm{C}, \mathrm{CHNH}_{3}{ }^{+}\right), 65.1$ (1C, C-18), $66.8\left(\mathrm{C}-\mathrm{CH}_{2 \mathrm{Glyc}}\right)$, 69.8 (1C, C-10), 73.9 (1C, C-11), 77.0 (4C, CH-Ala, C$2^{\prime}$ ). MALDIMS (negative mode): $m / z 1664.8[\mathrm{M}-\mathrm{H}]^{-}$, $1595.2[(\mathrm{M}-\mathrm{Ala})-\mathrm{H}]^{-}$.

3.18. $s n$-Glycero-hydroxyphosphoryl-(2-O-D-alanyl-snglycero)-hydroxyphosphoryl-(2-O-D-alanyl-sn-glycero)hydroxyphosphoryl-(2-O-D-alanyl-sn-glycero)-hydroxyphosphoryl-(2-O-D-alanyl-sn-glycero)-hydroxyphospho-

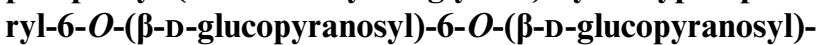
1,2-di- $O$-myristoyl-sn-glycerol (3)

Compound 3 was synthesized following the same procedure as described for compound 1. Compound 21 (174 mg, $0.048 \mathrm{mmol}$ ); $\mathrm{CH}_{2} \mathrm{Cl}_{2} / \mathrm{MeOH} / \mathrm{H}_{2} \mathrm{O}$ (7.5:7.5: 1.5, $6 \mathrm{~mL})$. Compound $3(24 \mathrm{mg}, 26 \%)$ was obtained as a white powder. ${ }^{1} \mathrm{H}$ NMR $\left(600 \mathrm{MHz}, \mathrm{D}_{2} \mathrm{O}\right): \delta 0.77-$ $0.93(\mathrm{~m}, 6 \mathrm{H}, \mathrm{Me}), 1.12-1.43\left(\mathrm{~m}, 40 \mathrm{H}, \mathrm{CH}_{2}\right.$-chain), 1.51-1.71 (m, 16H, Ala-Me, $\left.\mathrm{COCH}_{2} \mathrm{CH}_{2} \mathrm{R}\right), 2.23-2.48$ (m, $\left.4 \mathrm{H}, \mathrm{COCH}_{2} \mathrm{CH}_{2} \mathrm{R}\right), 3.23-4.62(\mathrm{~m}, 48 \mathrm{H}), 5.28-5.45$ (m, 5H, CH-Ala, $\left.2^{\prime}-\mathrm{H}\right) .{ }^{13} \mathrm{C}$ NMR (150.9 MHz, $\left.\mathrm{D}_{2} \mathrm{O}\right)$ : $\delta 16.6(2 \mathrm{C}, \mathrm{Me}), 18.1$ (4C, Ala-Me), 24.0 (2C, $\left.\mathrm{COCH}_{2} \mathrm{CH}_{2} \mathrm{R}\right), 25.7 / 33.0 / 35.1$ (20C, $\mathrm{CH}_{2}$-chain), 52.0 $\left(4 \mathrm{C}, \mathrm{CHNH}_{3}{ }^{+}\right), 65.1(1 \mathrm{C}, \mathrm{C}-18), 66.8\left(\mathrm{C}-\mathrm{CH}_{2 \mathrm{Glyc}}\right)$, 69.8 (1C, C-16), 73.9 (1C, C-17), 77.0 (5C, CH-Ala, C$2^{\prime}$ ). MALDIMS (negative mode): $m / z$ 1890.4 $[\mathrm{M}-\mathrm{H}]^{-}$.

3.19. $s n$-Glycero-hydroxyphosphoryl-(2-O-D-alanyl-snglycero)-hydroxyphosphoryl-(2-O-D-alanyl-sn-glycero)hydroxyphosphoryl-(2-O-D-alanyl-sn-glycero)-hydroxyphosphoryl-(2-O-D-alanyl-sn-glycero)-hydroxyphosphoryl-(2-O-D-alanyl-sn-glycero)-hydroxyphosphoryl-6- $O$ ( $\beta$-D-glucopyranosyl)-6- $O$-( $\beta$-D-glucopyranosyl)-1,2-di$O$-myristoyl-sn-glycerol (4)

Compound 4 was synthesized following the same procedure as described for compound 1. Compound 24 (83 mg, $0.021 \mathrm{mmol}$ ); $\mathrm{CH}_{2} \mathrm{Cl}_{2} / \mathrm{MeOH} / \mathrm{H}_{2} \mathrm{O}$ (7.5:7.5:1.5, $6 \mathrm{~mL})$. Compound $4(12 \mathrm{mg}, 28 \%)$ was obtained as a white powder. ${ }^{1} \mathrm{H}$ NMR $\left(600 \mathrm{MHz}, \mathrm{D}_{2} \mathrm{O}\right): \delta 0.77-0.93$ (m, 6H, Me), 1.12-1.43 (m, 40H, $\mathrm{CH}_{2}$-chain), 1.51$1.71\left(\mathrm{~m}, 19 \mathrm{H}\right.$, Ala-Me, $\left.\mathrm{COCH}_{2} \mathrm{CH}_{2} \mathrm{R}\right), 2.23-2.48(\mathrm{~m}$, $\left.4 \mathrm{H}, \mathrm{COCH}_{2} \mathrm{CH}_{2} \mathrm{R}\right), 3.23-4.62(\mathrm{~m}, 48 \mathrm{H}), 5.28-5.45(\mathrm{~m}$, $\left.6 \mathrm{H}, \mathrm{CH}-\mathrm{Ala}, 2^{\prime}-\mathrm{H}\right) .{ }^{13} \mathrm{C}$ NMR $\left(150.9 \mathrm{MHz}, \mathrm{D}_{2} \mathrm{O}\right): \delta$ $16.6(2 \mathrm{C}, \mathrm{Me}), \quad 18.1$ (5C, Ala-Me), 24.0 (2C, $\mathrm{COCH}_{2} \mathrm{CH}_{2} \mathrm{R}$ ), 25.7/33.0/35.1 (20C, $\mathrm{CH}_{2}$-chain), 52.0 $\left(5 \mathrm{C}, \mathrm{CHNH}_{3}^{+}\right), 65.1(1 \mathrm{C}, \mathrm{C}-18), 66.8\left(\mathrm{C}-\mathrm{CH}_{2 \mathrm{Glyc}}\right)$,
69.8 (1C, C-16), 73.9 (1C, C-17), 77.0 (6C, CH-Ala, C$\left.2^{\prime}\right)$. MALDIMS (negative mode): $\mathrm{m} / z 2114.5[\mathrm{M}-\mathrm{H}]^{-}$, $2041.9[(\mathrm{M}-\mathrm{Ala})-\mathrm{H}]^{-}$.

\section{Acknowledgement}

This work was supported by the Deutsche Forschungsgemeinschaft and the Fonds der Chemischen Industrie.

\section{References}

1. Westphal, O.; Lüderitz, O.; Keiderling, W. Zentralbl. Bakteriol. Parasitenkd. Infektionskr. Hyg. 1952, 158, 152 160.

2. Wright, S. D.; Ramos, R. A.; Tobias, P. S.; Ulevitch, R. J.; Mathison, J. C. Science 1990, 249, 1431-1433.

3. Shimazu, R.; Akashi, S.; Ogata, H.; Nagai, Y.; Fukudome, K.; Miyake, K.; Kimoto, M. J. Exp. Med. 1999, 189, 1777-1782.

4. Nagai, Y.; Akashi, S.; Nagafuku, M.; Ogata, M.; Iwakura, Y.; Akira, S.; Kitamura, T.; Kosugi, A.; Kimoto, M.; Miyake, K. Nat. Immunol. 2002, 3, 667-672.

5. Morath, S.; Geyer, A.; Hartung, T. J. Exp. Med. 2001, 193, 393-397.

6. Grangette, C.; Nutten, S.; Palumbo, E.; Morath, S.; Hermann, C.; Dewulf, J.; Pot, B.; Hartung, T.; Hols, P.; Mercenier, A. Proc. Natl. Acad. Sci. U.S.A. 2005, 102, 10321-10326.

7. Henneke, P.; Morath, S.; Uematsu, S.; Weichert, S.; Pfitzenmaier, M.; Takeuchi, O.; Muller, A.; Poyart, C.; Akira, S.; Berner, R.; Teti, G.; Geyer, A.; Hartung, T.; Trieu-Cuot, P.; Kasper, D. L.; Golenbock, D. T. $J$. Immunol. 2005, 174, 6449-6455.

8. Morath, S.; Geyer, A.; Spreitzer, I.; Hermann, C.; Hartung, T. Infect. Immun. 2002, 70, 938-944.

9. Lehner, M. D.; Morath, S.; Michelsen, K. S.; Schumann, R. R.; Hartung, T. J. Immunol. 2001, 166, 5161-5167.

10. Hermann, C.; Spreitzer, I.; Schröder, N. W.; Morath, S.; Lehner, M. D.; Fischer, W.; Schutt, C.; Schumann, R. R.; Hartung, T. Eur. J. Immunol. 2002, 32, 541-551.

11. Schröder, N. W.; Morath, S.; Alexander, C.; Hamann, L.; Hartung, T.; Zähringer, U.; Gobel, U. B.; Weber, J. R.; Schumann, R. R. J. Biol. Chem. 2003, 278, 1558715594.

12. Hoebe, K.; Georgel, P.; Rutschmann, S.; Du, X.; Mudd, S.; Crozat, K.; Sovath, S.; Shamel, L.; Hartung, T.; Zähringer, U.; Beutler, B. Nature 2005, 433, 523-527.

13. Morath, S.; Stadelmaier, A.; Geyer, A.; Schmidt, R. R.; Hartung, T. J. Exp. Med. 2002, 195, 1635-1640.

14. Stadelmaier, A.; Morath, S.; Hartung, T.; Schmidt, R. R. Angew. Chem. 2003, 115, 945-949; . Angew. Chem., Int. Ed. 2003, 42, 916-920.

15. Stadelmaier, A.; Figueroa-Perez, I.; Deininger, S.; von Aulock, S.; Hartung, T.; Schmidt, R. R. Bioorg. Med. Chem. 2006, 14, 6239-6254.

16. Deininger, S.; Stadelmaier, A.; von Aulock, S.; Morath, S.; Schmidt, R. R.; Hartung, T. J. Immunol. 2003, 170, 4134-4138.

17. Figueroa-Perez, I.; Stadelmaier, A.; Morath, S.; Hartung, T.; Schmidt, R. R. Tetrahedron: Asymmetry 2005, 16, 493506. 
18. Classon, J.; Garegg, P. J.; Samuelson, B. Acta Chem. Scand., Ser. B 1984, B38, 419-422; Johansson, R.; Samuelson, B. J. Chem. Soc., Perkin Trans. 1 1984, 2371-2374.

19. Coste, J.; Le Nguyen, D.; Castro, B. Tetrahedron Lett. 1990, 31, 205-208.
20. Fieser, L. F.; Fieser, M. In Reagents for Organic Synthesis; Wiley: New York, 1967; Vol. 1, p 782.

21. Deininger, S.; Figueroa-Perez, I.; Sigel, S.; Stadelmaier, A.; Schmidt, R. R.; Hartung, T.; von Aulock, S., in preparation. 